\title{
Vertical Slot Convection: a Linear Study
}

\author{
A. McAllister, ${ }^{a)}$ R. Steinolfson,${ }^{b)}$ and T. Tajima \\ Institute for Fusion Studies \\ The University of Texas at Austin \\ Austin, Texas 78712
}

\begin{abstract}
We investigate the linear stability properties of fluid convection in a vertical slot. We use a Fourier-Chebychev decomposition to set up the linear eigenvalue problems for the Vertical Slot Convection and Benard problems, and solved for the eigenvalues, neutral stability curves, and critical point values of the Grashof number, $G$, and the wavenumber, $\alpha$. Plots of the real and imaginary parts of the eigenvalues as functions of $G$ and $\alpha$ are given for a wide range of the Prandtl number, $\operatorname{Pr}$, and special note is made of the complex mode that becomes linearly unstable above $\operatorname{Pr} \sim 12.5$. A discussion comparing different special cases facilitates the physical understanding of the VSC equations, especially the interaction of the shear-flow and buoyancy induced physics.

Making use of the real and imaginary eigenvalues and the phase properties of the eigenmodes, we categorize the eigenmodes. We find that the mode structure becomes progressively simpler with increasing $\mathrm{Pr}$, with the greatest complexity in the mid ranges where the terms in the heat equation are of roughly the same size.
\end{abstract}

a) Currently at the University of Tokyo, Tokyo, Japan

${ }^{b}$ Currently at Southwest Research Institute, San Antonio, TX 


\section{An Introduction}

When the driving stress on a fluid body in a gravitational field is a temperature gradient the result is buoyancy driven thermal convection. The classic arena for studying thermal convection is the Benard problem in which a horizontal fluid layer is heated from below. ${ }^{1} \mathrm{~A}$ related problem consists of a vertical fluid slab, such that the thermal gradient is perpendicular, rather than parallel, to the gravitational field. This change in geometry introduces a background 1-D shear flow and increases the complexity of the problem. We call this the Vertical Slot Convection (VSC) problem.

The initial experimental workers on this problem where interested in the heat transport across the slot (insulation and cooling problems), ${ }^{2,3}$ while the theorists focused on the breakdown of the initial shear flow as the temperature difference is increased. ${ }^{4,5}$ While there has been continued interest in the engineering applications of this problem, ${ }^{6,7,8}$ with experimental work at progressively higher temperature gradients, ${ }^{9,10}$ there has been growing interest in this problem from a theoretical viewpoint as a nonlinear dynamics problem. ${ }^{11}$ Beyond this we are also interested in its applications to other areas of physics, especially the fact that many aspects of $2-\mathrm{D}$ convective fow problems have direct parallels in plasma physics. ${ }^{12}$

Although experimental work has been done mostly in low and medium aspect ratio slots $(h=10-60)$ it has been found that the end affects of the slots do not propagate affect more than $10 \%$ at either end ${ }^{13}$ so that $h=\infty$ theoretical and numerical studies are believed to apply to much of the height of a tall thin slot. ${ }^{10,14}$ As this situation allows for exact solutions of the basic shear flow much of the theoretical work has been done for $h=\infty$ ratio slots. Chait and Korpela ${ }^{15}$ give a nice discussion of the advantages and disadvantages of taking $h$ infinite in a simulation, including the need to fix the wavenumber.

The modern theoretical literature dates to a paper by Batchelor ${ }^{5}$ and focused in the 
mid 60 's, ${ }^{16,17,19}$ and the early 70 's, ${ }^{19,20,21,22}$ on making use of various limits to expand the equations and obtain the linear stability characteristics of the 1-D background shear flow. This is, however, a laborious process and yields results of limited accuracy, so as computers have become more powerful they have been used to study this problem.

MacGregor and Emery 23 give a good review of early computational work, although their study is on low aspect ratio $h=1,10$ experimental and sinnulation results. Medium $h$ numerical and simulation results include some of those in Refs. 6,9 , and $2 \dot{\dot{x}}(h=15)$. Raithby and Wong ${ }^{10}$ give results for $h$ up to 80 , but these high $h$ results are computationally expensive. With more powerful computers the linear results were more exact, ${ }^{25}$ but there are still computational advantages to the infinite aspect formulation.

Besides the aspect the slot geometry the physics depends on the type of fluid in use, as reflected in the value of the Prandtl number, Pr. Experimenters have used quite a wide ranges of fluids including air: $\operatorname{Pr} \sim .7^{3,23}$ water: $\operatorname{Pr} \sim 7 ;^{26.7}$ oils: $\operatorname{Pr}=25^{28} \operatorname{Pr}=480,^{27}$ $\operatorname{Pr} \sim 1000,{ }^{26,28}$ and $\operatorname{Pr}=12,500 .{ }^{27}$ The theoreticians have also made use $c_{\text {. } ~}$ he limit $\operatorname{Pr} \quad 0$ to decouple the heat equation from the equation of motion..$^{29,30}$

Early reviews which lean to experimental work are found in Eckert and Carlson, ${ }^{3}$ Elder, ${ }^{13}$ Vest and Arpaci. ${ }^{28}$ Pepper and Harris ${ }^{6}$ cover earlier computational work and Raithby and Wong $^{10}$ treat finite $h$ simulations. Good general reviews can be found in Bergholtz, ${ }^{31}$ Patterson and Imberger, ${ }^{3 \_}$and Lee and Korpela. ${ }^{24}$

This paper presents an introduction to the VSC problem in the form of a comprehensive linear study with respect to the background shear flow, and as such is a mixture of original calculations and a review of previous work. We chose to work with $h=\infty$ and explore the whole $(G, \alpha, \operatorname{Pr})$ parameter space (to $G=10,000, \alpha=10.0$, and $\operatorname{Pr}=0-1000$ ). We will, however, focus mostly on low and mid $\operatorname{Pr}$, up to $\operatorname{Pr} \sim 10$. The main thrust is to improve on the accuracy of the eigenvalues, to map their structure, and to produce and categorize the VSC eigenmodes in preparation for their use in later nonlinear studies. To bring out the 
physics behind each of the terms and modes of the full set of equations, we will examine a hierarchy of truncated models of both the VSC and Benard cases. Comparing the results from all cases serves to bring a fuller understanding on this system of equations.

\section{The VSC Model}

The Vertical Slot Convection (VSC) problem is illustrated in Fig. 1. The cold wall, at $x=-L$, is held at temperature $T_{1}$ and the hot wall, at $x=+L$, at temperature $T_{2}$. The gravitational force is in the negative $z$-direction, $\mathbf{g}=(0,0,-1)$.

The equations we use to treat this problem are derived from the general hydrodynamic equations $^{1,33}$ by applying the Boussinesq approximation. ${ }^{34}$ The incompressable equation of continuity is:

$$
\nabla \cdot \mathbf{u}=0
$$

where $\mathbf{u}$ is the fluid velocity.

Assuming that the pressure driven density variation is a negligible the equation of motion is just the Navier-Stokes equation:

$$
\rho_{\circ} \frac{D}{D t} \mathbf{u}=-\nabla P+\mu \nabla^{2} \mathbf{u}+\rho^{\prime} \mathbf{g}
$$

where $\rho$ is the density, $\rho^{\prime}$ in the thermal density variation, $\frac{D}{D t}=\frac{\partial}{\partial t}+\mathbf{u} \cdot \nabla$ is the convective derivative, $P$ is the pressure variation over the hydrostatic background, and $\mu$ is the coefficient of kinematic viscosity.

Ignoring viscous dissipation, the incompressable equation of heat conduction is:

$$
\rho_{D} C_{p} \frac{D}{D t} T=k \nabla^{2} T
$$

where $T \equiv T_{\text {tot }}-\left(T_{1}+T_{2}\right) / 2$ is the deviation from the mean base temperature $T_{o}, k$ is the coefficient of heat conduction and $C_{p}$ is the specific heat at constant pressure. We also define 
$\Delta T \equiv\left(T_{2}-T_{1}\right) / 2$ so the temperature gradient is equal to $\Delta T / L$. An appropriate equation of state is

$$
\rho=\rho_{o}(1-\alpha T)
$$

where $\alpha$ is the volume expansion coefficient and we continue to ignore all pressure driven density variations.

The VSC configuration does not have a heat conductive solution as its ground state. Instead for any nonzero temperature gradient $(\Delta T>0)$ the ground sta $: \mathrm{e}$ is a single convective cell with fluid rising along the hot wall and falling along the cold will. For a slot with an infinite aspect ratio there is a 1-D steady-state solution describing this flow:

$$
\begin{aligned}
& W(x)=W_{\circ} \frac{x}{6}\left(1-x^{2}\right) \\
& T(x)=\Delta T x
\end{aligned}
$$

where $W_{o}=\alpha g L^{2} \Delta T / \nu$, is the maximum vertical velocity of this flow $\left(\nu=\mu / \rho_{0}\right)$. Found $r_{\text {; }}$ balancing the viscous shear force and the buoyancy force, it serves as an appropriate reference velocity.

We renormalize the VSC equations using $W_{0}, \Delta T, L$, and $\rho_{0}$ :

$$
\begin{gathered}
\nabla \cdot \mathbf{u}=0, \\
\frac{D}{D t} \mathbf{u}=-\nabla P+\frac{1}{G} \nabla^{2} \mathbf{u},+\frac{1}{G} T \bar{z} \\
\frac{D}{D t} T=\frac{1}{\operatorname{Pr} G} \nabla^{2} T .
\end{gathered}
$$

The equations are now written in terms of two dimensionless parameters, the Grashof number and the Prandtl number:

The Grashof number is: $G=\alpha g L^{3} \Delta T / \nu^{2}=W_{0} L / \nu$, which shows explicitly the dependence 
on the driving temperature gradient and is related to the Rayleigh number by $R a=G P r$. The definition of $G$ in terms of $W_{0}$ shows it can be regarded as a Reynolds' number, which is meaningful at small Prandtl numbers when the thermal equation decouples from the equation of motion.

The Prandtl number is: $\operatorname{Pr}=\nu / \kappa$, where $\kappa=k / C_{p} \rho_{o}$, which is the ratio of the relative responsiveness of the fluid to kinetic and thermal stresses. This is a key parameter that couples thermal gradients into the equations of motion, and, for a given $G$ regulates the strength of coupling between the main equations.

It is well known that for shear flow between parallel walls the initial instabilities are two dimensional, ${ }^{4}$ allowing us to recast the equation of motion in terms of a stream function and a vorticity. Choosing to work in the $x-z$ plane we take the $y$ component of the curl of Eq. (7):

$$
\frac{\partial}{\partial t} \omega+\frac{\partial(\omega, \psi)}{\partial(x, z)}=-\frac{1}{G} \frac{\partial T}{\partial x}+\frac{1}{G} \nabla^{2} \omega
$$

where the stream function $\psi$ is defined by

$$
v_{x}=\frac{\partial \psi}{\partial z} \quad v_{z}=-\frac{\partial \psi}{\partial x},
$$

the vorticity $\omega$ by

$$
\boldsymbol{\omega}=\nabla \times \mathbf{u}
$$

and the general form of the Jacobian is

$$
\frac{\partial(\phi, \psi)}{\partial(x, z)} \equiv \frac{\partial \phi}{\partial x} \frac{\partial \psi}{\partial z}-\frac{\partial \phi}{\partial z} \frac{\partial \psi}{\partial x}
$$

Similarly we can rewrite the equation of heat conduction as:

$$
\frac{\partial}{\partial t} T+\frac{\partial(T, \psi)}{\partial(x, z)}-\frac{1}{\operatorname{Pr} G} \nabla^{2} T=0
$$

We note that the continuity equation (1) is now identically satisfied but has in practice been 
replaced by the relationship between $\omega$ and $\psi$ :

$$
\omega=\left(\frac{\partial^{2}}{\partial x^{2}}+\frac{\partial^{2}}{\partial z^{2}}\right) \psi .
$$

Thus our model will consist of Eqs. (9), (13), and (14).

In the normalized units the boundary conditions are given by

$$
\begin{array}{cll}
T= \pm 1 & \text { at } x= \pm 1 & \text { for all } z, \\
\psi=\text { a constant } & \text { at } x= \pm 1 & \text { for all } z, \\
\partial_{x} \psi=0 & \text { at } x= \pm 1 & \text { for all } z .
\end{array}
$$

The constant value for $\psi$ is assumed to be zero, and the definition (10) guarantees that this models rigid, no-slip boundaries.

In this work we treat the infinite aspect ratio case which applies in the limit of very tall, thin, slots, and to the central regions of shorter slots, whenever end effects can be ignored. The top and bottom boundaries are thus taken to be periodic, a condition automatically maintained by a vertical Fourier decomposition.

\section{The Linear Problem}

To derive the linear equations we follow the standard technique, ${ }^{1}$ linearizing Eqs. (9) and (13) by separating the variables into a 'background' part plus a perturbation, i.e. $\phi=\phi_{b}+\hat{\phi}$.

Only the Jacobian cross-terms introduce a dependence on the particular state with respect to which the equations have been linearized. For the cases we are interested in, the steadystate base flows are independent of $z$, and the $z$ partial derivatives of $\psi_{b}, \omega_{b}$, and $T_{b}$ are zero giving the linearization of Eqs. (9) and (13) as:

$$
\begin{gathered}
\frac{\partial \widehat{\omega}}{\partial t}=-\frac{\partial \omega_{b}}{\partial x} \frac{\partial \hat{\psi}}{\partial z}+\frac{\partial \psi_{b}}{\partial x} \frac{\partial \widehat{\omega}}{\partial z}-\frac{1}{G} \frac{\partial \hat{T}}{\partial x}+\frac{1}{G} \nabla^{2} \hat{\omega}, \\
\frac{\partial \hat{T}}{\partial t}=-\frac{\partial T_{b}}{\partial x} \frac{\partial \hat{\psi}}{\partial z}+\frac{\partial \psi_{b}}{\partial x} \frac{\partial \hat{T}}{\partial z}+\frac{1}{G P r} \nabla^{2} \hat{T} .
\end{gathered}
$$

Note that because the stability question and the longer-term evolution of perturbations are separate issues, we can impose 'background' flows as part of the initial states by including them in the linearized Jacobian terms. 
We assume the following form for the perturbation fields:

$$
\widehat{\psi}(x, z, t)=\hat{\psi}(x) \exp (\lambda+i \gamma) t \exp i \alpha z
$$

where $\lambda, \gamma$, and $\alpha$ are real, and similarly for $\hat{\omega}(x, z, t)$ and $\hat{T}(x, z, t)$.

Inserting these into Eqs. (15) and (16) we get

$$
\begin{gathered}
\lambda \hat{\psi}(x)=\frac{1}{G}\left(\partial_{x}^{2}-\alpha^{2}\right) \hat{\omega}(x)-i \alpha \partial_{x}\left(\omega_{b}\right) \hat{\psi}(x)+i \alpha \partial_{x}\left(\psi_{b}\right) \widehat{\omega}(x)-\frac{1}{G} \partial_{x} T, \\
\lambda \hat{T}(x)=\frac{1}{\operatorname{Pr} G}\left(\partial_{x}^{2}-\alpha^{2}\right) \hat{T}(x)-i \alpha \partial_{x}\left(T_{b}\right) \hat{\psi}(x)+i \alpha \partial_{x}\left(\psi_{b}\right) \hat{T}(x),
\end{gathered}
$$

with constraints:

$$
\left(\partial_{x}^{2}-\alpha^{2}\right) \widehat{\psi}(x)-\widehat{\omega}(x)=0
$$

and

$$
\widehat{T}( \pm 1)=\hat{\psi}( \pm 1)=\partial_{x} \hat{\psi}( \pm 1)=0
$$

We now replace each $x$ dependent perturbation with a series of Chebychev polynomials $T_{n}(x)$, such that

$$
\hat{\psi}(x)=\sum_{n=0}^{N^{\prime \prime}} T_{n}(x) \psi_{n}
$$

and similarly for $\widehat{\omega}(x)$ and $\hat{T}(x)$ (using Orzag's notation. ${ }^{35,36}$ ) Finally, we replace the fixed functions of $x$ representing the base solution and the remaining partial derivatives with matrix operators. We thereby formulate a complex generalized eigenvalue problem that can be solved numerically for $\lambda$, and the functions $\hat{\psi}(x), \widehat{\omega}(x)$, and $\widehat{T}(x) .{ }^{36}$

\section{A. The linear hierarchy}

We can now introduce a hierarchical sequence of linearized models, starting with the NavierStokes equations and building up to the full VSC equations, the analysis of which illuminates the physics behind the modes of the full equations and indicates clearly which terms each mode depends on. We include the Benard problem and a special case with a background 
shear flow as the comparison elucidates the symmetry dependance of the VSC modes. The results for the models outlined below are presented in Sec. 4.

There are two characteristics of the eigenvalues and the eigenmodes that we particularly want to examine here. These are the reality or complexity of the eigenvalues and the phase relationships of the eigenmodes. The key to sorting this out lies in the mode derivatives. Our equations are of the form

$$
\lambda \hat{\phi}=\mathbf{A} \hat{\phi}+\mathbf{B} \widehat{\Xi}
$$

where $\mathbf{A}$ and $\mathbf{B}$ are differential operators, $\hat{\phi}$ is one of our variables, and $\widehat{\Xi}$ represents a vector of the other variables in the system. These two operators determine, respectively, the reality or complexity of the eigenvalue $\lambda$ and the phase relations of $\hat{\phi}$ with the variables represented by $\hat{\boldsymbol{\Xi}}$. If $\mathbf{A}$ is real, the eigenvalue is real. This happens whenever the operator includes only even numbers of derivatives.

With perturbations of the form given in $\mathrm{Eq}$. (17) we can see that each spatial derivative will pull down a factor of $i$ (consider a complex exponential representation for the Chebychev polynomials). The number of derivatives in $\mathbf{B}$ acting on one of the variables in $\widehat{\underline{\Xi}}$ serves to produce a relative phase equal to that number times $\frac{\pi}{2}$. Thus, for instance, Eq. (11) implies that the vorticity and the stream function fields are always exactly opposite in phase.

\section{The Navier-Stokes equation}

We consider first the Navier-Stokes equation linearized about a null initial state

$$
\frac{\partial \hat{\omega}}{\partial t}=\frac{1}{G} \nabla^{2} \widehat{\omega} .
$$

This is a simple Laplacian, which can be solved using Fourier analysis to show that the real eigenvalue $\lambda$ is always less than zero and $\gamma$ is zero (see Ref. 36, chp. 3). These solutions are stable (decaying) real modes (real $\lambda$ and $\gamma=0$ ). The steady-state solution is stable and depends on the boundary conditions. 
Physically this term describes viscous dissipation through which an arbitrary flow relaxes, in the absence of additional driving terms, to a uniformly motionless fluid ( $\hat{\omega}$ assumed uniform along the boundary).

The Navier-Stokes equation with a shear flow initial state, which includes the Jacobian terms, has essentially the same solutions as the $P r=0$ case given next. It is not, however, self-consistent (as it is missing the thermal gradient term necessary for the 1-D solution) whereas the $\operatorname{Pr}=0$ case is.

\section{The $\operatorname{Pr}=0$ limit}

This case is both the $\operatorname{Pr}=0$ limit $(\kappa \rightarrow \infty$ while the $\nu$ remains finite) of the full VSC problem, and the equivalent to the Navier-Stokes case with the VSC 1-D solution as the initial condition. The relevant equations are

$$
\begin{gathered}
\frac{\partial \widehat{\omega}}{\partial t}=-\frac{\partial \omega_{b}}{\partial x} \frac{\partial \hat{y}_{i}}{\partial z}+\frac{\partial \psi_{b}}{\partial x} \frac{\partial \widehat{\omega}}{\partial z}-\frac{1}{G} \frac{\partial \widehat{T}}{\partial x}+\frac{1}{G} \nabla^{2} \widehat{\omega}, \\
\frac{\partial \hat{T}}{\partial t}=\frac{1}{G P r} \nabla^{2} \widehat{T} .
\end{gathered}
$$

The addition of the temperature Laplacian in Eq. (25) does not affect the kinetic variable $(\hat{\psi}$ and $\widehat{\omega}$ ) solutions, as its diffusive nature drives $\hat{T} \rightarrow 0$. This, in turn, means that for any significant $G$ the thermal gradient term in the equation of motion (24) is essentially zero and the kinetic variable solutions are the same as those for the equivalent NavierStokes equation. When Eq. (24) is linearized without the gradient term it becomes the OrrSommerfeld equation. ${ }^{28,19,291}$ In the full equations there are often kinetic variable gradients driving the fluid motions as well. This special case is useful for checking which motions are purely isothermal as opposed to those driven by thermal gradients.

\footnotetext{
${ }^{1}$ The Orr-Sommerfeld equation can also be derived in the limit $G \rightarrow \infty, 16,20$ in which case the buoyancy term is dropped while the viscous term is kept, although it is also proceeded by $\frac{1}{G}$. We also note that with a different basic state it describes linearized Poiseuille flow. ${ }^{37}$
} 


\section{The zero-velocity initial state}

This case is the VSC equivalent of the standard Benard problem. It is also the first in which thermal gralients are coupled into the equations of motion. The initial state consists of a thermal gradient, $T=x$, but has null kinetic fields. The reduced equations are

$$
\begin{aligned}
& \frac{\partial \widehat{\omega}}{\partial t}=-\frac{1}{G} \frac{\partial \hat{T}}{\partial x}+\frac{1}{G} \nabla^{2} \widehat{\omega}, \\
& \frac{\partial \hat{T}}{\partial t}=-\frac{\partial \hat{\psi}}{\partial z}+\frac{1}{G P r} \nabla^{2} \widehat{T} .
\end{aligned}
$$

It is only self-consistent for $G=0$, since it is only for $G=0$ that the null initial state is a solution of the unperturbed form of these equations, due to the otherwise nonzero value of the term $\frac{1}{G} \frac{\partial T_{b}}{\partial x}$. This initial state, although unstable for all $G>0$, is equivalent to the Benard initial equilibrium, and serves as the base state from which the 1-D equilibrium bifurcates. In general this case describes the establishment of the 1-D shear flow after a sudden switching on of the temperature gradient. In this regard it is suited to yield information about the transients involved in the establishment of such a flow (see Ivey, Ref. 38).

The phase structure found in this case holds for the rest of our case studies as well. The thermal gradient term in Eq. (26) leads to a phase difference of $\frac{\pi}{2}$ between the temperature and the vorticity, while the gradient of the stream function appearing in Eq. (27) leads to a similar difference (with a few exceptions, see Sec. 4.6.5) between the stream function and the temperature. Thus the phase of the temperature leads that of the vorticity by $\frac{\pi}{2}$ and lags that of the stream function by the same amount. The modes in this case are real (real $\lambda, \gamma=0)$ as there are no contributions from the Jacobian terms to the operator $\mathbf{A}$.

\section{The Benard problem cases}

The classic Benard problem, where the initial state is a motionless fluid with a conductive temperature profile, $T=x$, is also represented by Eqs. (24) and (25), except that the partial 
of $\hat{T}$ is taken with respect to $z$ (rather than $x$ ) and thus is zero for $T_{b}=x$. The null kinetic solution is, in this case, a consistent initial condition. The Benard case has real modes and the same relative phasing as the zero-velocity initial state case, though the difference in the gradient term may lead to differences in the overall mode phase at large $\mathrm{Pr}$.

We will also refer to a special case where the Benard equations are expanded around the 1-D solution (Eqs. (5) and (6)), so that we have added a shear flow to the thermal gradient in the initial conditions. Although this background state is not a solution to the full Benard equations (with the usual boundary conditions), it is the best horizontal comparison to the full VSC case, indicating which modes are purely shear driven and which depend as well on the perpendicularity of the gravitational force.

The equations for this case are the same as those for 1 ? : VSC problem (also expanded about the 1-D solution), Eqs. (15) and (16), but with the $\mathbf{x}$ gradient of $\widehat{T}$ replaced with a $\mathbf{z}$ gradient. This case, and the full VSC problem, both yield a mixture of real and complex $(\gamma \neq 0)$ modes. The relative phasing is again the same as in Sec. 3.1.3.

\section{The Linear Computational Results}

We now turn to the results of our linear study of the related VSC and Benard convection problems. These are based on a computer code that solves the eigenvalue problem posed by linearizing the governing equations, as outlined in Sec. 3 (also Ref. 36, Appendix C1). We present graphic and quantitative results for the full Vertical Slot Problem, followed by discussion in the context of supplementary results for the various special cases, which serve to clarify the significance of the VSC findings. Together, these results iliustrate points made in the presentation of the governing equations and provide specific new qualitative and quantitative measures of the effects of the different terms and parameters in the full set of equations. Comparison with previous results provides a good test of our numerical method. 


\section{A. The general VSC eigenvalue structure}

We start, in Fig. 2a, with the eigenvalues for the VSC proi n plotted versus the Grashof number for a given Prandtl number and wavenumber, chosen here as $\operatorname{Pr}=7.5$ (water) and $\alpha=1.383$ (the corresponding linearly critical wavenumber). The real parts of the largest eigenvalues are shown in Fig. 2a, where the horizontal line near the top of the plot marks $\lambda=0$, indicating that most of these modes are decaying modes that would appear physically as short-lived transients. ${ }^{2}$

At low $G$ most of the modes are real modes, meaning that the eigenvalue is real $(\gamma=0)$; however as $\widetilde{C}$ increases these combine two at a time to form pairs of complex modes with eigenvalues of the form $\lambda \pm i \gamma .{ }^{17}$ Physically this represents the change from stationary modes, which may grow or decay but do noi move in the slot, to pairs of traveling modes that travel up and down the hot and cold walls of the slot respectively.

Two $\mathrm{n} \rightarrow \mathrm{s}$ are of specific interest. The first is the sole stationary mode to persist as $G$ increases, becorning unstable at $G=491.8(\operatorname{Pr}=7.5)$ it is the main growing for all Prandtl numbers less than $\operatorname{Pr} \sim 12.5$. The second is the top complex mode which at $\operatorname{Pr}=7.5$ and for $G>150$ has a small, almost constant, negative real part to its eigenvalue. This mode is the primary transient for $\operatorname{Pr}<12.5$.

At larger Prandtl numbers the real part of this complex mode's eigenvalue, $\lambda_{c}$, becomes positive for some values of $G$ and $\alpha$. Above $\operatorname{Pr} \sim 12.5 \lambda_{c}$, becomes positive at a value of $G$ that is less than the critical value for the real mode so that there the critical modes are traveling modes. In Fig. $2 \mathrm{~b}$ we show the eigenmode structure at $\operatorname{Pr}=50$. Here we can see again the pairing of real modes as complex pairs. The top complex mode becomes unstable for roughly the range $70<G<440$ while the stationary mode can be seen rising across all

\footnotetext{
${ }^{2}$ A test with an increased number of modes showed that at larger $G$ only the real mode and the top six complex modes remain the same while at lower $G$ not more that the top dozen real or complex modes are unaltered.
} 
the complex modes, becoming unstable for $G>600$. As first observed by Birikh, et al. ${ }^{22}$ this real mode arises from complex modes that in turn form from pairing real modes. Birikh's group also pointed out that the small real part of the eigenvalue of the unstable complex mode limits it to large aspect slots, if the slot is too short the mode cannot grow before it reaches the top of the slot.

\section{B. The neutral stability curve}

Cornbining Eqs. (18) and (19) to eliminate $\hat{\psi}$ or $\hat{T}$ and setting $\lambda=0$ one obtains a fourth order partial differential equation which may be solved numerically to yield the neutral stability curve, $G_{c}(\alpha)$, for fixed $\operatorname{Pr}$ (our results, however, are based on searching for $\lambda=0$ with the eigenvalue solver). This curve separates the unstable modes $\left(\lambda_{\max }>0\right)$ and the stable modes $\left(\lambda_{\max }<0\right)$. Figure 3 shows the neutral stability curve for $\operatorname{Pr}=7.5$. Rudakov ${ }^{17}$ seems to be the first to have published such curves, sketches for $\operatorname{Pr} \leq 0.2$ and $G \leq 500$. Vest and Arpaci $^{28}$ present one for general $\operatorname{Pr}$ that reaches larger $G$, and Mizushima and Gotoh ${ }^{39}$ show one for $\operatorname{Pr}=7.5$ that ours matches well. The shape of this curve is essentially unchanged for all $\operatorname{Pr}<12.5$, moving slightly up and down in $G$ as $\operatorname{Pr}$ varies. At high $\operatorname{Pr}$ there is a new unstable complex mode (see Sec. 4.4.1) but the part of the neutral curve contributed by the statione $y$ mode bas essentially the same shape. Mizushima ${ }^{40}$ shows neutral curves for high $\operatorname{Pr}$ that drop significantly. However they are also wider at large $G$, suggesting insufficient resolution. Our study of mode truncation at lower $\operatorname{Pr},{ }^{36}$ indicates that $G_{c}$ is sharply reduced when there are too few modes.

Thus for low and mid $\operatorname{Pr}$ number regimes the vertical slot is linearly unstable to stationary modes with a wavenumber less than 2.0 at $G=1000$ and not more than 2.5 at high $G$ (in units of $L$ ). This upper limit on $\alpha$ holds for all $\operatorname{Pr}<1000$, where the traveling mode is unstable up to $\alpha \sim 2.7$ (see also Fig. 7). Thus the wavelength of any unstable mode must be greater than $\sim 2.5$, while the most unstable modes have an aspect ratio of $\sim 1.26$. 
There is also an upper limit on the wavelength as for $\alpha \rightarrow 0$, the system is stable for all $G$. Calculation of the mean flow as a function of $x$ for various wavenumbers at a fixed Grashof number ${ }^{41}$ shows that it is maximally reduced when the wavenumber is in the range $1.3-1.9$ so that a wavenumber is selected that will minimize the mean flow. The Prandtl number independence is a sign of the kits nature of these shear driven modes.

The stationary mode neut: stability curve is nearly but not quite symmetric around the line through the critical wavenumber at $\alpha=1.38348$, as it rises more steeply on the large $\alpha$ side. The critical Grashof number is $G=491.8187$. These values agree well with previous published results. Vest $\&$ Arpaci $^{28}$ gave values for a general $\operatorname{Pr}$ as $G=492.5$ and $\alpha=1.325$ respectively. Ruth ${ }^{25}$ gave the most accurate values published to date, of $G_{c}=491.776$ and $\alpha_{c}=1.3835$. Mizushima \& Gotoh ${ }^{39}$ gave $G=492.3$ at $\alpha=1.414$, which is a point on the neutral stability curve, but is not the critical point. In recent studies Fujimura and Mizushima ${ }^{42}$ have obtained results of a high order of accuracy, with which our results agree to five decimal places in $\alpha_{c}$ and differing slightly in the third place for $G_{c}$ (for more critical values see subsection 4.3 .

To test the numerical stability of these results we performed a series of neutral stability curve calculations varying the number of Chebychev modes, $N$. For $\operatorname{Pr}=7.5$ reducing $N$ causes $\alpha_{c}$ to increase while $G_{c}$ decreases (at $N=8 \alpha_{c}=1.65$ and $G_{c}=318.0$ ). The increase in $\alpha_{c}$ with decreasing $N$ reflects a general tendency for all curves and contours of $\lambda(G, \alpha)$ to spread towards larger $\alpha$ when the number of modes is insufficient to resolve the equations. This is especially true at high $G$ and high $\operatorname{Pr}$, reflecting the dwindling influence of the diffusion terms when the number of modes is too small. When $N$ is small the curves also drop in $G$ and for $N<12$ there appear new modes at high $\alpha$. Similarly Rudakov ${ }^{18}$ shows a case at $\operatorname{Pr}=10$ where reducing the number of modes will cause eigenvalues to become positive artificially.

On the other hand the values at 32 and 64 differ by not more than .001 , and in general, 
for $\operatorname{Pr}$ up to about 10.0, 32 modes seems to be sufficient unless extremely precise quantitative results are needed. For larger $P r$, however, the number of modes needed to properly resolve the equations increases rapidly. By $P r=1000$ even 64 modes are too few to prevent severe spreading of the neutral curves.

\section{Critical point sweeps vs. the Prandtl number}

Varying the Prandtl number, we find that although the qualitative form of the stationary mode neutral stability curve changes very little, there is quantitative variation, especially in $G$. The changes in the critical Grashof and wavenumbers for the real stationary mode as $\mathrm{Pr}$ varies from $\operatorname{Pr}=0.001$ to $P r=100$ are presented in Fig. 4. There are asymptotic regimes at low and high $P r$, where the dissipative and the nonlinear terms dominate, while in the central region there is competition between them.

These plots are in good agreement with similar ones in Rudakov, ${ }^{18}$ Ruth, ${ }^{25}$ and by Fujimura and Mizushima. ${ }^{42}$ Our results at specific values of $\operatorname{Pr}$ are also in good agreement with previous results; at $\operatorname{Pr}=0$, where $\alpha_{c}=1.34414$ and $G_{c}=495.6284,{ }^{28,41}$ at $\operatorname{Pr}=0.7$ (air) where $\alpha_{c}=1.4049$ and $G_{c}=502.5889,,^{28,24}$ and at $\operatorname{Pr}=6.7$ (also water) $\alpha_{c}=1.3836$ and $G_{c}=491.7479 .43$ We will return to discuss the correlation of the Prandtl number variation of the critical values with the physics of the modes when we discuss the VSC eigenmodes (see Sec. 4.6.5).

\section{D. $\lambda(\alpha, G)$ and $\gamma(\alpha, G)$}

To classify the modes that form solutions to the VSC problem we plot the maximum real eigenvalue $\lambda_{\max }$ as a function of $G$ and $\alpha$. In Fig. 5 we show a sequence of contour plots of $\lambda_{\max }(G, \alpha)$ at selected Prandtl numbers. The solid lines indicate positive values and therefore unstable regions, while the dashed lines are used for negative values or stable regions $(\lambda=0$ is the first dashed line). The main feature in all these cases is the unstable real mode at low 
$\alpha$. The eigenvalues for this mode remain relatively unchanged over a wide range of Prandtl numbers, especially at large $G$. Aside from the real mode the others are all complex, and for $\operatorname{Pr}<12.5$ they are all stable. Above $\operatorname{Pr} \sim 12.5$ there is an unstable complex mode at low $G$, low $\alpha$ which can be seen in the bottom two frames of Fig. 5 . We treat this separately in Sec. 4.4.1.

At $\operatorname{Pr}=0.001$ there is a change at approximately $\alpha=2.4$ to a series of complex modes that fill up the high $\alpha$ region. The complexity of the contours here arises from a patchwork of different modes, each largest in a different region of the parameter space. As the Prandtl number increases this patchwork is replaced by a single complex mode, which fans out from the vertical boundary with the real mode. For $\operatorname{Pr}>0.7$ this mode is the least negative at high $\alpha$ for all $G$.

While the plot of $\lambda(G, \alpha)$ shows clearly the most important physical property, the stability of the system, and gives the rates of growth or decay, it is not so clear what the range of each mode is and of what type it is. In order to explore the nature of the different eigenmodes further and clarify the their characters, we use col_. lots of two more functions.

The imaginary part of the eigenvalue, $\gamma(G, \alpha)$, is the mode frequency and is related to the phase velocity of the traveling modes, $v_{p}=\gamma / \alpha$. In Fig. 6 we show contour plots of $\gamma(G, \alpha)$. From the various values of $\operatorname{Pr}$ in Fig. 6 we see that the clear division of real and complex modes at low $\alpha$ starts breaking down about $\operatorname{Pr}=0.5$ when a complex mode first appears at low $G$ and $\alpha \sim 1.2$. As $\operatorname{Pr}$ increases, this presence grows and for $\operatorname{Pr}>\sim 2.0$ there is a complex mode at low $G$ for all $\alpha$ except those near zero. Also noticeable in this figure are the discontinuities in $\gamma$ that break the $(G, \alpha)$ plane into regions. Comparison with Fig. 5 shows that these discontinuities coincide with the abrupt slope changes seen in the contours of $\lambda$ and examination of the eigenmodes shows that they are caused by the change from one rnode to another with a different value of $\gamma$. Lastly, as $\operatorname{Pr}$ increases into the middle range where the effect of the nonlinear terms begins to be felt, there is a sweeping out towards 
higher $\alpha$ of one of these discontinuities that is related to the process by which a single mode comes to dominate in the whole complex part of the $(G, \alpha)$ plane.

\section{The high $\operatorname{Pr}$ complex mode}

We now turn to discuss the complex mode that shows up subcritically at about $\operatorname{Pr}=0.5$. As $\operatorname{Pr}$ increases, this traveling mode $\mathrm{e}^{26,44}$ becomes unstable, starting at high $G$ and then at progressively lower $G$ until, at $\operatorname{Pr}=12.4542$ when $G_{c}=492.056$, the critical number for this complex mode (at $\alpha_{c}=0.3424$ ) is equal to that for the stationary real mode (at $\alpha_{c}=1.38317$, these figures are from Fujimura and Mizushima. ${ }^{42}$ ) As $\operatorname{Pr}$ increases further, the complex mode becomes unstable for increasingly small $G$ and increasingly large $\alpha$ (see also Ref. 22). The critical Grashof number drops with increasing Prandtl number, reaching 15.7 at $\operatorname{Pr}=1000\left(\right.$ Elder $^{26}$ gives $\sim 20$, Vest \& Arpaci, ${ }^{28}$ 19.5. Our estimate is with $N=100$ ). The initial wavenumber for the traveling mode, which initially appears along the $\alpha=0$ axis, is at much lower $\alpha$ than that for the stationary mode. As $\operatorname{Pr}$ increases, however, the mode curls around under the stationary mode (see Figs. 5 and 7) and $\alpha_{c}$ for the travelirig mode grows until it is in the same range as $\alpha_{c}$ for the real mode (Vest \& Arpaci $^{28}$ give $\alpha=.925$ at $\operatorname{Pr}=1000$ but our data indicate a value of $\alpha=1.2$ there). These changes in $G_{c}$ and $\alpha_{c}$ for the complex mode are much greater than those for the real mode [Fig. 4] $]^{29,22}$

In Fig. 7 we show in detail the $\lambda(G, \alpha)$ contours at $\operatorname{Pr}=12.5,15$, and 100. The complex mode, rather than replacing the real mode, fills the space around it at low $\alpha$ and low $G$, and has generally smaller growth rates than the real mode. For $G=100$ there is an upper boundary in $G$ (below the value at which the stationary mode in turn becomes unstable) at which the mode becomes stable again. At some wavenumbers there is a direct exchange of stability, at others a gap in $G$. Only at small $\alpha$ does the complex mode persist at high $G$ and there only with a very small growth rate. 


\section{E. Other cases and discussion}

In order to clarify these results, we performed a similar analysis on the other cases described in Sec. 3.1 above. By comparing results with those for the full problem we can often see the physical consequences of the different terms in the full equations.

The Navier-Stokes Case: The results of the Navier-Stokes equations with no background shear flow should be compared to the $P r=0$ limit for the Benard case rather than the VSC, unless one imposes a sheared flow by starting with the 1-D solution for $\hat{\psi}$ and $\widehat{\omega}$. This is an entirely stable system, all modes being real with negative $\lambda$.

The $\operatorname{Pr}=0$ Limit: This limiting case illustrates which modes are driven by the kinetic terms (those involving $\hat{\psi}$ and $\widehat{\omega}$ ), modes which are the result of the interaction the Jacobian and the damping terms in the equations of motion. Assuming a background flow of the form of the 1-D solution to the full equations gives a self-consistent solution to the $\mathrm{Pr}=0$ equations, with a conductive temperature solution, $T=x$, that influences the equations of motion through the $-\frac{1}{G} \partial_{x} T$ term, even if it is only a constant. In the case of the Navier-Stokes equations alone we get the same results but only by starting with a sheared background flow.

This demonstrates that the min stationary mode is primarily driven by the central shearing of the 1-D solution without any direct input from the thermal gradient. As we increase the Prandtl number (from 0 ) this situation is 'perturbed' by the stronger coupling to the thermal gradients. 


\section{The Benard Problem:}

Although the nonlinear equations for this problem are nearly identical to the VSC equations, the change in coupling between them changes the symmetry of the problem with significant effects. The simplification of the linear physics can be related to two factors. The first is that the 'ground state' now corresponds to a motionless fluid, so that there is no initial background flow and no initial shearing.

Figure 8 shows the eigenvalues for the Benard problem. These are all real, stationary modes as we expected (see Sec. 3.1). The first positive mode peaks at $G \sim 75$ and thereafter decreases, while others become positive as well. The critical point at $\operatorname{Pr}=7.5$ is found to be $G_{c}=14.2313$ and $\alpha_{c}=1.55816$, which is in good agreement with Chandrashekar. ${ }^{1}$

For the Benard case the critical wavenumber is constant in $\operatorname{Pr}$, while $G$ varies inversely. This implies that the critical Rayleigh number $R a_{c}=\operatorname{Pr}^{*} G_{c}$ is also constant in $\operatorname{Pr}$, as it is known to be. In fact the whole solution for $\lambda(G, \alpha)$ scales with $\operatorname{Pr}$, so that the contour plot for $\operatorname{Pr}=10$ shown in Fig. 9 can be scaled in $G$ to give that for any value of $\operatorname{Pr}$. Although the scales are very different from Fig. 5, comparison shows that the Benard case is unstable for much larger values of $\alpha$ as well as for much smaller values of $G$. We see that the result of losing the Jacobian (the convective) terms leads to exclusively real modes and a very simple variation with $\mathrm{Pr}$.

Benard with Shear Flow: A second difference between the Benard and the VSC problems lies in their respective symmetries. In order to do a more realistic comparison and display the result of changing the configuration from a vertical to a horizontal slot, we assumed a background shear flow in the Benard configuration. Physically this can be applied to many problems in fluid pipe flows or atmospheric physics. ${ }^{45}$ The results in this case are essentially the same as those for the $\mathrm{VSC}$ problem at low $\mathrm{Pr}$, again indicating that these modes depend 
on the shearing in the center of the slot and not on the thermal equation or how it couples to the equations of motion.

Plots of the critical values against the Prandtl number, Fig. 10, show that in a qualitative way the values for $\operatorname{Pr}<0.1$ are similar to those for the VSC problem (see Fig. 4). At higher $\operatorname{Pr}$, however, these curves are quite different. The critical Grashof number, $G_{c}$, falls farther and never rises again, approaching an asymptotic limit of $\sim 305$ at large $\operatorname{Pr}$. The critical wavenumber plot rises much higher at the initial peak (near $\operatorname{Pr}=0.1$ ) and only slightly at the second (around $\operatorname{Pr}=1.0$ ). This is the inverse of the VSC case. Also in the present case the large $\operatorname{Pr}$ value $\alpha_{c}=1.327$ is slightly lower than the low $\operatorname{Pr}$ limit, rather than quite a bit higher, as was true for the VSC case.

Figure 11 shows that at low $\operatorname{Pr}$ the mode structure is also same as that for the VSC configuration (see Figs. 5 and 6). With increasing $\operatorname{Pr}$ the complex modes again reduce to a single dominant mode, which curls around under the stationary mode at higher $\operatorname{Pr}$, but it does not become unstable. Therefore, while one might expect to see stationary modes develop in this system, there is nothing to break the horizontal symmetry along the slot and thus lead to traveling modes.

These comparisons indicate that the VSC stationary mode arises from the center-slot shear and is driven only indirectly by the thermal gradient. This gradient gives rise to the background shear flow and is therefore the indirect source of energy for the stationary mode. The development of a supercritical complex, or traveling mode, at larger $P r$ is seen to depend on the specific geometry of the buoyancy force, rather than the interaction of the thermal gradient with the shear flow.

The Zero-state VSC Case: The results of this case parallel those for the Benard problem, as was expected; although $\alpha_{c}=1.266$ is slightly smaller, it is again constant, while $G_{c}$ is inversely proportional to $\mathrm{Pr}$, although roughly 4 times larger at any given value of $\mathrm{Pr}$. 
As in the Benard case, the plot for $\lambda(G, \alpha)$ shown in Fig. 12 can be scaled in $G$ to give the correct plot for any value of $P r$. The qualitative form of this plot is similar to that of Fig. 9, although the magnitude of $\lambda$ is generally smaller. This implies that in the growth of background shear flow in the VSC case occurs with a longer time scale than does the growth of cells in the Bernard configuration."

Comparing the graphs of $\lambda(G, \alpha)$ for the sheared cases with those that have a motionless initial state, we can see that the shearing stabilizes the high $\alpha$ modes, limiting the wavenumber to $\alpha \leq 2.5$. Where there is no shear flow the wavenumber can be much larger, especially at high $G$, and thus we can have much smaller cell aspect ratios. The shear stretches out any secondary flow causing it to have a longer wavelength and a smaller wavenumber. ${ }^{46}$

\section{F. Eigenmode zoology}

We now come to the eigenmodes themselves. As was the case with the eigenvalues, we work, at any given set of values for the parameters $\{G, \operatorname{Pr}, \alpha\}$, with the mode that has the largest value of $\lambda$. Thus we talk of different modes at different values of the parameters $G$ and $\alpha$, even though mathematically they may all be present over the whole $G$ - $\alpha$ plain. We will also usually refer to a complex pair of eigenmodes as a single mode, except when it is relevant to compare them to each other. Thus at any given set of parameters there is only one 'mode' under consideration.

It has been possible to define properties that can be used to classify the modes and we start with an outline of these properties. There follows a general enumeration of modes, though it should be kept in mind that although mode properties can be used in combination to differentiate and define 'modes', these 'modes' can vary with changing $G$ and $\alpha$. There are instances both of abrupt changes in these properties from one 'mode' to the next, and also cases of a slow transformation with no distinguishable discontinuity. The modes are arranged here so as to move from the simplest ones to the more complex, with reference 
made to the hierarchy of equations discussed in Sec. 3.1.

All mode plots are contour plots of the respective field with a width equal to that of the slot and a height equal to a single vertical wavelength. Thus although they are plotted as squares, they represent aspect ratios of $\lambda / 2=\pi / \alpha$.

\section{Analytic tools}

\section{The Horizontal Wavelength}

The 'horizontal wavelength' is a qualitative measure based on the number of positive and negative vortices across the stream function $(\hat{\psi})$ eigenfield. A single vortex filling the slot (e.g. Fig. 21b) thus has a 'wavelength' of twice the slot width. There are also modes with 'wavelengths' equal to the slot width, one half the slot width, or even less. In the complex modes there are even modes with same sign vortices next to each other, however, we will still refer to the 'wavelength' even, though the cross section is more like two half waves than a single full wave.

Figure 21b shows, a real mode with a double slot wavelength and Fig. 22a a complex mode that has a half slot wavelength, a "frequency doubled" mode (right). The complex mode (one half of a pair) is considered as only filling half the slot width, even though it tails all the way across. Relative to these stream function fields the vorticity fields always have a shorter wavelength, while the temperature fields are always of the same wavelength (there is one exceptional case at $\operatorname{Pr}=1.0$ of a small group of modes whose temperature field also has half-slot wavelength).

There is a wavelength halving process in which the vortices split into two halves horizontally and then shift out of phase (vertically) with each other as the parameters vary. When they are sufficiently out of phase (on the order of $\frac{\pi}{2}$ ) the mode appears to have doubled its horizontal frequency.

When we talk of "stretching" or "pulling back" of modes, this refers to changes in the 
horizontal distribution of the mode in the slot in which the fields pull back towards the walls, stretching out in the middle of the slot. There is a general, though not absolute, tendency for modes to pull back with higher $G$ and higher $\alpha$, reflecting the evolution towards boundary layer physics in these regimes. At large $\operatorname{Pr}$ the thermal fields pull back but the kinetic fieids remain the same, or expand slightly.

Phase Properties: There are, especially at low $P r$, quite a variety of different complex modes and even of real modes, when overall mode phase is taken into consideration. In trying to sort these out, we have made use of several properties, based on a measure of the 'phase' of an eigenfield. This phase is derived from the ratio of the real and imaginary parts of the largest element of the mode's vertical Fourier transform. The "overall phase" of an eigenmode (applying to all three fields) is defined from the temperature field, the only one that always has a single clear peak horizontally. We adopted this analysis after noticing that, with small variation, there is a strong tendency for the modes to have half-integral $\pi$ overall phases. In Fig. 14 has a phase of zero $(\hat{t})$, while Fig. 21d illustrates a phase of $\frac{\pi}{2}$. Multiplying these fields by -1 gives fields with phases of $-\frac{\pi}{2}$ and $\pi$.

The "relative phases" are the phase differences between the different fields of the same mode (for complex modes we measure the $\lambda+i \gamma$ half of each pair). As discussed in Sec. 3.1.3 we expect a fixed pattern of relative phase differences, and, indeed, the relative phases of the kinetic variables always differ by $\pi$. While for most modes the phase difference between the $\widehat{\psi}$ and $\widehat{T}$ fields is $\frac{\pi}{2}$, there are a few where there is no difference. These are called "in-phase" modes.

A third type of phase comparison involves taking one half of a pair of complex fields and flipping it about the vertical and horizontal centerlines of a cell one wavelength high and the width of the slot (rotating it by 180 degrees about the cell's center point). When we compare the rotated mode with its partner, it is found to be always either in phase or out of 
phase by half a wavelength. We refer to this as a mode's "flipped phase." In all cases either the $\hat{\psi}$ and $\widehat{\omega}$ pairs are in phase and the $\widehat{T}$ fields not, or vice versa. In Fig. 13 we show a pair of $\psi$ fields that are out of phase with each other (the left-hand half is always the $\lambda+i \gamma$ one).

\section{The Navier-Stokes case}

The rıode structure for the Navier-Stokes equations with no background flow, $W_{b}=0$, is simple, as there are only real stable modes and no $\widehat{T}$ field. The $\hat{\psi}$ fields all have a horizontal wavelength of twice the slot width, while the vorticity has a wavelength that is half this. Except for their phases these fields are qualitatively the same as those for the Benard case shown in Fig. 14. Shifting the Benard kinetic fields up by a quarter frame $\left(\frac{\pi}{2}\right)$ gives the Navier-Stokes fields, such that $\hat{\psi}$ has a phase of zero and $\widehat{\omega}$ a phase of $\pi$. For $\alpha<6.67$ the Navier-Stokes modes all have the same phase, but for $\alpha>6.67$ they change by $\pi$. In this region the magnitude of $\widehat{\omega}$ is proportional to $\hat{\psi}$ rather than varying inversely, due to a minus sign connected with the change in phase.

As $\alpha$ increases there is a stretching out of the fields. This is most noticeable in the vorticity where the quarter wavelength features near the walls narrow and the central features widen to fill nearly the whole slot. There is no similar dependency on $G$.

\section{The Benard problem}

As there is still $\mathrm{L}_{\mathrm{O}} \mathrm{o}$ background shear flow, the mode structure in the Benard case is nearly the same as the Navier-Stokes case described above but with the addition of an equally simple temperature field (all Benard field plots are turned vertical rather than being horizontal). Again all the modes are real, although they are no longer uniformly stable. The kinetic fields' wavelengths are the same as above, while the $\hat{T}$ fields have the same wavelength as the $\hat{\psi}$ fields. In Fig. 14 we show a typical example of the main Benard mode. These fields can be well approximated with the proper combination of sinusoidal functions as is done 
in the well-known Lorenz formulation. ${ }^{47}$ The horizontal stretching out of this mode has the same variation as the Navier-Stokes case.

The basic mode has an overall phase of zero. For $\operatorname{Pr} \leq 1.0$, however, there are some low $G$ and high $\alpha$ modes with different overall phases. At $\operatorname{Pr}=1.0$ they appear only at very small $G$, however, for lower $\operatorname{Pr}$ they cover more of the $(G, \alpha)$ plane and below about $\operatorname{Pr}=0.5$ maintain the pattern seen in Fig. 15, which shows the phases of the Benard temperature eigenmodes for $\operatorname{Pr}=0.1$, where the nonzero phase exist at values of $G(\alpha)$ about ten times as large as those reached at $\operatorname{Pr}=1.0$. For higher $\operatorname{Pr}$ these modes are apparently completely absent.

The low $G$, low and medium $\alpha$ mode has an overall phase of $\frac{\pi}{2}$, while the high $\alpha$ mode has an overall phase of $-\frac{\pi}{2}$. This pattern is identical at $\operatorname{Pr}=0.01$ and does not scale with $\operatorname{Pr}$, unlike the values of $\lambda(G, \alpha)$. The transitions from one mode to the next are sharp discontinuities.

\section{The zero-state case}

The eigenmodes in this case have a nonzero horizontal phase gradient, or pitch (determined by the ratio of the odd over the even horizontal components of the mode,$^{25}$ ) and appear tilted. Although there is no background vertical flow here, the buoyancy term that couples the vertical gravity with the horizontal thermal gradient causes the fluid near the warm wall (on the right) to rise as it circulates across the slot, while the fluid near the cool wall (on the left) falls as it circulates. This leads to a component with odd horizontal symmetry and tilts the modes, as seen in Fig. 16, where the dramatic tilt is due to the large value of $G$. As $G \rightarrow 0$ the tilt also goes to zero. Comparing with the Benard modes one finds that aside from pitch the modes are qualitatively the same.

The low $\operatorname{Pr}$ phase structure seen in the Benard case is also found in the zero-state problem. It is the exactly the same as that in Fig. 15 for all $\operatorname{Pr}$ up to $\sim 0.5$ above which it 
starts retreating to smaller $G$, until it disappears between $\operatorname{Pr}=0.75$ and $\operatorname{Pr}=1.0$, slightly lower than in the Benard case. On the other hand at larger $\operatorname{Pr}$ (and probably for very large $G$ and $\alpha$ even at smaller $\operatorname{Pr}$ ), there are new modes with a phase of $-\frac{\pi}{2}$. Unlike the low $\operatorname{Pr}$ nonzero phase modes, the high $\operatorname{Pr}$ phase structure does scale with $\operatorname{Pr}$, like the eigenvalues.

In Fig. 17 we show the zero-state phase diagram at $\operatorname{Pr}=7.5$ with a band of $-\frac{\pi}{2}$ phase modes. Scaling $G$ inversely with $\operatorname{Pr}$ will give the structure for other values of $P r$. If the pitch of the modes is plotted, the phase boundaries of Fig. 17 are found to lie along equal pitch lines. These curves drop rapidly at low $\alpha$, then bottom out and turn upwards again, rising with increasing speed at larger $\alpha$. A line can be drawn from the origin through the turning points of these equal pitch lines which is roughly parabolic $\left(G \propto \alpha^{2}\right)$. The width of this parabola varies as $\operatorname{Pr}$. The pitch varies smoothly as $G$ and $\alpha$ are increased or decreased with no discontinuity at the phase discontinuities.

\section{The VSC problem}

With the inclusion of the background shear flow we obtain complex solutions as well as real ones. This corresponds traveling modes, paired complex modes that ride on the shear flow background, traveling up the warm side and down the cool side of the slot.

The VSC modes may be generally categorized using Figs. 5 and 6 above for $\lambda$ and $\gamma$, supplemented by Figs. 18 and 19 below. These show the overall phases and the structure of the relative and flipped phases describ.2d in Sec. 4.6.1. This combination of properties gives a fairly clear description of the modes.

Figure 18 shows the regions of the four main phases; zero (vertical stripes), $\frac{\pi}{2}$ (positive definite stripes;, $\pi$ (negative definite stripes), and $-\frac{\pi}{2}$ (horizontal stripes). There are also regions at low $\mathrm{P}_{t}$ where the phases are not half integrals of $\pi$, these are indicated by the overlay regions that are especially frequent around $\operatorname{Pr}=0.03$.

At low $\operatorname{Pr}$ there is a profusion of modes with different phases. In the $\operatorname{Pr}=0.001$ plots 
a zero-phase mode can be seen near the real/complex boundary at high $G$. With increasing $\operatorname{Pr}$, this phase spreads out first to low $\alpha$ and then to high $\alpha$ until, by $\operatorname{Pr} \sim 0.76$, the whole $(G, \alpha)$ plain is dominated by zero-phase modes. This expansion correlates with the movement of the discontinuities in the $\gamma(G, \alpha)$ plots (see Fig. 6), while the low $G$ and high $\alpha$ structure for $\operatorname{Pr}=0.1$ to $\sim 0.7$ is similar to that seen in the Benard and zero-state cases (see Fig. 15).

Figure 19 illustrates the internal phase properties of the complex modes. The complex modes can in all cases be divided into those which, when flipped, are $\pi$ out of phase in the temperature (negative definite stripes), most numerous at small $\operatorname{Pr}$, and the majority, for which the kinetic variables are out of phase (vertical stripes). At $\operatorname{Pr} \sim 0.5$ and above all modes have this property.

Half wavelength modes are indicated by the horizontal stripes. All the real modes and those complex modes that appear for $\alpha<\sim 2.4$ at all $\operatorname{Pr}$ have a horizontal wavelength that is equal to the width of the slot. For low Pr up to about 0.1 this is true for all modes, except a small vertical strip that corresponds to a complex mode at $G>5000$ and $\alpha \sim 2.5$. This mode, which lies on the real/complex boundary, has a wavelength equal to half the width of the slot. Above $\operatorname{Pr} \sim 0.1$ it spreads out to higher $\alpha$ and lower $G$, until at larger $\operatorname{Pr}$ all modes for $\alpha>2.0$ have this property. The low $\alpha$ unstable complex modes that appear at high $\operatorname{Pr}$ em do not have this property.

Finally the in-phase modes are shown in positive definite stripes. These modes appear in a band to the right (higher $\alpha$ ) of the wavelength halved modes and are swept out in front of them as they unfold with increasing $\operatorname{Pr}$.

There is a general tendency for modes and properties that appear at high $G$ along the real/complex boundary at low $\operatorname{Pr}$ to sweep out and down as $\operatorname{Pr}$ increases, first the zero phase, then the in-phase band and finally the wavelength halving property.

A good method for summarizing the various mode properties is to plot their ranges 
against the Prandtl number (see Fig. 20), with the critical numbers plotted for reference. Our results agree well with Ruth's study of the odd and even field components whose ratio gives the field pitch. ${ }^{25}$ The low- $\alpha$ expansion of the zero-phase mode starts near $\operatorname{Pr}=0.01$, as soon as the effects of the nonlinear terms in the thermal equation begin to be felt, pulling the critical values away from their low $\operatorname{Pr}$ values. The jumble of high $G$, mid- $\alpha$ complex modes also begins to change at this point and the pitch of the critical real mode begins to decrease. At $\operatorname{Pr} \sim 0.03$ the in-phase modes first appear and the zero-phase mode begins its sweep to the high- $\alpha$ side. At $\operatorname{Pr}=0.07$ the low- $\alpha$ modes are all zero-phase and the pitch of the critical mode temperature field (the odd temperature component) goes through zero and starts to grow with the opposite sign. At $\operatorname{Pr}=0.1$, the $G_{c}$ minimum, the reversed pitch is equal in magnitude to its low $\operatorname{Pr}$ value, and the expansion of the half-slot wavelength complex modes begins. As $G_{c}$ increases again, the temperature field pitch continues to increase until at $\operatorname{Pr}=0.5$, where $G_{c}$ peaks, it has the same magnitude as that of the stream function field.

Overall the pitch of the critical mode stream function is essentially independent of $P r$, while that of the temperature changes continuously. For $\operatorname{Pr}>0.5$ it is larger in magnitude than the kinetic pitch, but it is inverted, sloping up to the hot wall. The $G_{c}$ peak also coincides with the completion of the zero-phase mode expansion, and the disappearance of the in-phase and complex modes that are "out of phase" in the temperature. The low $\alpha$ complex modes start to appear subcritically at $\operatorname{Pr} \sim 0.5$, although they do not become unstable until $\operatorname{Pr} \sim$ 12.5. Lastly the zero-phase mode expansion is complete at $\operatorname{Pr} \sim 0.75$ the peak in $\alpha_{c}$ and the half wavelength expansion is complete at $\operatorname{Pr}=1.0$.

One exception to our agreement with $\mathrm{Ruth},{ }^{25}$ is that he found a rapid reversal in sign of all rixode components at the $\operatorname{Pr} \sim 2.0$ minimum in $\alpha_{c}$. This should change the overall mode phase by a factor of $\pi$, a change we do not see.

In summary we may describe three regimes; a low $\operatorname{Pr}$ regime, up to $\operatorname{Pr} \sim 0.01$, where the flows are essentially isothermal; a high $\operatorname{Pr}$ regime, above $\operatorname{Pr} \sim 0.75$ in which the thermal 
effects are fully coupled in and there is a single stationary and two complex modes, all with zero overall phase; and, in between, a transition regime where the shifting balance of terms leads to a series of changes in the modes with changing $\mathrm{Pr}$. This regime can be classified into two sub-regimes associated, respectively, with the decline and the rise of $G_{c}$, below and above $\operatorname{Pr}=0.1$.

Sample VSC modes: We conclude our linear results with some illustrative examples of specific modes demonstrating their range of form and indicating how to estimate the form of modes not shown explicitly. We start with the small $\operatorname{Pr}$ modes and work towards increasing $\operatorname{Pr}$ (see Ref. 36 for additional figures).

Pr=0.001: The typical real mode, shown in Fig. 21a, is similar the zero-state mode, but with an overall phase of $\pi$ and generally less pitch, as the background flow velocities reduce the relative magnitude of the buoyancy effect. We can also see the effect of the background flow on the form of the vorticity field. The only other mode (at low $P r$ ) is the vertical strip between the neutral stability curve $(\alpha \sim 2.0)$ and the real/complex boundary $(\alpha \sim 2.5)$ where the real modes have a reverse pitch. At low $G$ there is a continuity in form between these modes and the $\lambda-i \gamma$ modes at slightly higher $\alpha$.

Figures $21 \mathrm{~b}$ and $21 \mathrm{c}$ show the range of variation of the typical full-slot wavelength complex modes that are common at lower Prandtl number. Most complex modes, especially those near the real/complex boundary, have some reverse pitch due to the interaction of the background flow and the natural buoyancy. This effect decreases with increasing $\alpha$. Increasing $\alpha$ and/or $G$ causes the fields, which center on the line of maximum background velocity, to become more compact. This line moves closer to the walls at larger $G$.

The variations on these modes can be described by noticing that in many cases the mode fields seem to split along this line of maximum velocity. The boundary side and the 
interior sides seem to have phases and pitches that vary independently as the parameters are changed. We have already commented on the shifting relative phase of these parts that leads to the half wavelength modes. Often both parts have different pitches, thought the degree of difference varies widely (e.g. Fig. 22a).

In Fig. 21d we see a variation found near the real/complex boundary which has been drawn out vertically. The pitch is uniformly reversed, especially rare in the vorticity, which usually has reversed interior and normal exterior pitch as in Fig. 21b. The strong reverse pitch of the kinetic fields, fades at higher $\alpha$ and is only found at medium $G$.

There is exceptional another mode near the real/complex boundary, but at high $G$, that is drawn out in the other direction so that it has standard pitch. It has zero overall phase but more interestingly the $\hat{\psi}$ field makes this is a transition mode with ill-defined 'wavelength'. The $\hat{T}$ and $\hat{\omega}$ fields are similar to those of Fig. $21 \mathrm{~b}$, but the $\hat{\psi}$ field somewhere between that shown in Fig. 13 (or Fig. 22c) and that of Fig. 21d with strong inverse pitch. In fact, with a proper path in parameter space (and ignoring the shift from one phase to another) the mode in Fig. 21d changes smoothly into a half wavelength mode at $G=6000$ and $\alpha=2.75$.

The strength of the two sets of vortices in the stream function field (one along the wall and the other bounding the center-slot line) are not of equal strength, the interior one being stronger. This is in contrast to Fig. 13 above or Fig. 22a below.

Mid-Range $\operatorname{Pr}$ Special Cases: The preceding survey of modes at $\operatorname{Pr}=0.001$ gives a good summary of most of the fields encountered at larger $\operatorname{Pr}$ as well. There are, however, several special cases found for middle range $P r$ that should be mentioned. In general a look at the 'characteristics' of a mode as outlined above and reference to the examples given will yield a good idea of what it looks like. The one example of a completely different mode is shown in Fig. 22a. It is an example of a small group of modes at $\operatorname{Pr}=0.1$, for which the temperature as well as the $\psi$ field has half-slot wavelength. We mentioned above that for a middle range 
of $\operatorname{Pr}$, from perhaps 0.025 to 0.75 , there are a band of modes with a zero relative phase between the $T$ and $\psi$ modes. These modes can be imagined by shifting the interior parts of Fig. 22a downward, to yield full-slot wavelength fields.

Pr=7.5 Modes: In studying the VSC problem the Prandtl number for water is often chosen both for its intrinsic interest and as a good basis for comparison to previous work. It is high enough to be out of the mid range where all the complexities lie and thus for our purposes here serves to illustrate the typical high $\operatorname{Pr}$ modes.

There are essentially three types of modes, one real mode that is mostly unstable and two complex modes, one of which becomes unstable for slightly higher values of $\mathrm{Pr}$. In Fig. 22b we show the linearly critical mode $\left(\lambda=0.48 \times 10^{-7}\right)$ which agrees very well with previous results. ${ }^{18,21}$ This form is typical of the real modes for higher $\operatorname{Pr}$. The temperature field is confined to the center of the slot and has a strongly reversed pitch. The stream function field has two maxima, one on either side of a slight central minima. Ruth's study ${ }^{25}$ indicates that these changes in profile start from $\operatorname{Pr} \sim 0.5$. The central concentration of the temperature perturbation reflects the decreasing width of the full flow thermal boundary layers. Comparison with Fig. $21 \mathrm{a}$, coupled with the discussion of mode properties at the end of Sec. 4.6.5, allows one to extrapolate the form of the real modes for the whole middle range of $\operatorname{Pr}$. The differences in these two vorticity fields have more to do with the difference in $G$ than that in $\mathrm{Pr}$.

The two complex modes may be called 'low $\alpha$ ' and 'high $\alpha$ ' with the dividing line in the range $\alpha=2.0-2.5$. Even for parameters where these modes are stable, they will appear as transient pairs of upward and downward (right side and left side), traveling modes.

The high $\alpha$ mode, shown in Fig. 22c is a typical half-wavelength mode with kinetic flippedphase. 'The temperature field is an arrowhead type. Comparison with the $\operatorname{Pr}=0.001$ mode shown in Fig. 21b shows how the temperature field has pulled in to the wall while the kinetic 
boundary layer remains about the same (the wavenumber is slightly higher in Fig. 22c, contributing to the reduced width of both fields).

Finally the low $\alpha$ mode is the one that becomes unstable first at $\operatorname{Pr}>12.5$ (see Refs. 3, 6, 18, and 43). Here we show the complex critical mode pair at $\operatorname{Pr}=12.45$ where this mode has the same critical Grashof number as the real mode, Fig. 22d. At lower $\operatorname{Pr}$ has essentially the same form, although it holds more tightly to the lines of maximum $W_{b}$ as $\operatorname{Pr}$ increases. Although for lower $\operatorname{Pr}$ this mode is stable, linearly and nonlinearly, it can be seen as a transient. In fact, it forms the first transient seen in our nonlinear simulations. ${ }^{36,48}$

This figure also illustrates how a complex pair of modes would appear together in a simulation or experiment, however, at these specific values of $G$ and $\operatorname{Pr}$ one would expect to actually see the stationary mode as well and that there might be resonances between them.

\section{Summary}

We have thoroughly reviewed the properties of the solutions to the linear VSC problem and compared these with solutions of the Benard problem and several special cases, focusing on the interaction between the shear flow and buoyancy. In the process we present a systematic method of investigating a set of equations by looking at a series of special cases that serve to isolate the effects of the various terms. We have also derived some new diagnostics for describing the eigenmode solutions.

Our results check well against previous work and add considerably to the detailed knowledge of these solutions, especially the linear eigenmodes. These modes are of great use in our studies of the nonlinear VSC problem, ${ }^{48,49}$ where we use them as clean initial perturbations. We can also use our linear results to check the the resultant transients and initial growing modes produced by general perturbations of the nonlinear equations.

Having introduced the VSC problem in this paper, including its relation to the Benard problem etc., we will present the results of simulations of the full nonlinear VSC equations 
in a series of papers, several of which are already in preparation. ${ }^{\mathbf{4 8 , 4 9}}$

Beyond the considerable interest of this problem as an arena for studying nonlinear dynamics, we also plan to apply the results to various astrophysical problems. An example can be found in the upper solar convection zone, near the edges of sun spots or granular boundaries, where the accumulation of very strong vertical magnetic fields can induce a horizontal temperature gradient. ${ }^{50}$ In these cases we hope to eventually include magnetic fields as well, but might expect an enhancement of the traveling modes and a damping of the convective cells.

\section{Acknowledgments}

Work supported by NSF ATM9113576, NASA, and the U.S. Department of Energy, contract \#DE-FG05-80ET-53088. During the writing of this paper one of the authors (A.McA.) was supported by a JSPS post-doctoral fellowship. The computation was largely carried out at San Diego Super Computing Center. 


\section{References}

1. S. Chandrasekhar, Hydrodymanic and Hydromagnetic Stability, (Oxford University Press, Oxford, 1961).

2. W. Nusselt, V.D.I. Forsch. Arb. 63, 64, and 78 (!909).

3. E.R.G. Eckert and W.O. Carlson, Natural convection in an air layer enclosed between two vertical plates with different temperatures, Int. J. Heat Mass Trans. 2, 106-120 (1961).

4. H.B. Squire, On the stability of three-dimensional disturbances of viscous fluid flow between parallel walls, Proc. Roy. Soc. London, Ser. A 142,621-628 (1933).

5. G.K. Batchelor, Heat transfer by free convection across a closed cavity between vertical boundaries at different temperatures, Quart. Appl. Math. 12, 209-233 (1954).

6. D.W. Pepper and S.D. Harris, Numerical simulation of natural convection in clos 1 containers by a fully implicit method, J. Fluid Engin. 99, 649-656 (1977).

7. S.M. ElSherbiny, G.D. Raithby, and K.G.T. Hollands, Heat transfer by natural convection across vertical and inclined air layers, J. Heat Trans. 104, 96-1081 (1982).

8. G.D. Mallinson and G. De Vahl Davis, The method of the false transient for the solution of coupled elliptic equations, J. Comput. Phys. 12, 435-461 (1973).

9. M.E. Newell and F.W. Schmidt, Heat transfer by laminar natural convection within rectangular enclosures, J. Heat Trans. 92, 159-168 (1970).

10. G.D. Raithby and H.H. Wong, Heat transfer by natural convection across vertical air layers, J. Heat Trans. 104, 96-102 (1982). 
11. K. Fujimura and J. Mizushima, Truncation effect in a Fourier series expansion on the nonlinear evolution of disturbances in free convection between vertical parallel plates, Phys. Fluids 30, 2398-2400 (1988).

12. W. Horton, Nonlinear drift waves and transport in magnetized plasma, Phys. Rep. 192, 1-177 (1989).

13. J.W. Elder, Laminar free convection in a vertical slot, J. Fluid Mech. 23, 99-111 (1965).

14. K.G.T. Hollands and L. Konicek, Experimental study of the stability of differentially heated inclined air layers, Int. J. Heat Mass Trans. 16, 1467-1476 (1973).

15. A. Chait and S.A. Korpela, The secondary flow and its stability for natural convection in a tall vertical enclosure, J. Fluid Mech. 200, 189-216 (1989).

16. K. Gotoh and M. Satoh, The stability of a natural convection between two parallel vertical plates, J. Phys. Soc. Jpn. 21, 542-871 (1966).

17. R.N. Rudakov, On small perturbations of convective motion between vertical plates, PMM 30, 362-368 (1966).

18. R.N. Rudakov, Spectrum of perturbations and stability of convective motion between vertical plates, PMM 31, 349-355 (1967).

19. K. Gotoh and N. Ikeda, The stability of an unsteady convection between parallel vertical plates, J. Phys. Soc. Jpn. 30, 864-871 (1971).

20. K. Gotoh and N. Ikeda. Asymptotic solution of the instability problem of channel flows with antisymmetric velocity profile, J. Phys. Soc. Jpn. 32, 845-850 (1972). 
21. K. Gotoh and N. Ikeda, Secondary convection in a fluid between parallel vertical plates of different temperatures, J. Phys. Soc. Jpn. 33, 1697-1705 (1972).

22. R.V. Birikh, G.Z. Gershuni, E.M. Zhukhovitskii, and R.N. Rudakov, On oscillatory instability of plane-parallel convective motion in a verticl channel, PMM 36, 745-748 (1972).

23. R.K. MacGregor and A.F. Emery, Free convection through vertical plane layers moderate and high Prandtl number fluids, J. Heat Trans. 91, 391-403 (1969).

24. Y. Lee and S.A. Korpela, Multicellular natural convection in a vertical box, J. Fluid Mech. 126, 91-121 (1983).

25. D. Ruth, On the transition to transverse rolls in an infinite vertical fluid layer - a power series solution, Int. J. Heat Mass Trans. 22, 1199-1208 (1979).

26. J.W. Elder, Turbulent free convection in a vertical slot, J. Fluid Mech. 23, 99-111 (1965).

27. N. Seki, S. Fukusako, and H. Inaba, Visual observation of natural convective flow in a narrow vertical cavity, J. Fluid Mech. 84, 695-704 (1978).

28. C.M. Vest and V.S. Arpaci, The stability of natural convection in a vertical slot, J. Fluid Mech. 36, 1-15 (1969).

29. S.A. Korpela, D. Gozum, and C.B. Baxis, On the stability of the conduction regime of natural convection in a vertical slot, Int. J. Heat Mass Trans. 16, 1467-1476 (1973).

30. K. Fujimura and J. Mizushima, Nonlinear interaction of disturbances in free convection between vertical parallel plates, eds. R.W. Miksad, T.R. Akyla, T. Herbert, Nonlinear Wave Interactions in Fluids- AMD 87, 1987, pp. 123-130. 
31. R.F. Bergholz, Instability of steady natural convection in a vertical fluid layer, J. Fluid Mech. 84, 743-768 (1978).

32. J. Patterson and J. Imberger, Unsteady natural convection in a rectangular cavity, J. Fluid Mech. 100, 65-86 (1980).

33. L.D. Landau and E.M. Lifshitz, Fluid Mechanics (Pergamon Press, Oxford, 1987), 2nd ed.

34. D.J. Triton, Physical Fluid Dynamics (Van Nostrand, New York, 1977).

35. D. Gottlieb and S.A. Orzag, Nurnerical Analysis of Spectral Methods: Theory and Applications, (SIAM, Philadelphia, 1977).

36. A.McAllister, Numerical linear and nonlinear investigation of vertical slot convection, Ph.D thesis, The University of Texas at Austin, 1972.

37. J.T. Stuart, On the nonlinear mechanics of wave disturbances in stable and unstable parallel flows-part 1, the basic behavior in plane Poiseuille flow, J. Fluid Mech. 9, 353-370 (1960).

38. G.N. Ivey, Experiments on transient natural convection in a cavity, J. Fluid Mech. 144, 389-401 (1984).

39. J. Mizushima and K. Gotoh, Nonlinear evolution of the disturbance in a natural convection induced in a vertical fluid layer, J. Phys. Soc. Jpn. 52, 1206-1214 (1983).

40. J. Mizushima, Equilibrium solution of the secondary convection in a vertical fluid layer between two parallel plates, Fluid Dyn. Res. 5, 289-299 (1990).

41. M. Nagata and F.H. Busse, Three-dimensional tertiary motions in a plane shear layer, J. Fluid Mech. 135, 1-26 (1983). 
42. K. Fujimura and J. Mizushima, Nonlinear equilibrium solutions for traveling waves in a free convection between vertical parallel plates, Eur. J. Mech. B/Fluids, 10, no. 2 suppl., 25-30 (1991).

43. J.E. Hart, Stability of the flow in a differentially heated inclined box, J. Fluid Mech. 47, 547-576 (1971).

44. A.E. Gill, The boundary-layer regime for convection in a rectangular cavity, J. Fluid Mech. 26, 515-536 (1966).

45. D.J. Stensrud, The expected branching solution: preferred wavelengths and orientations, ed. H.N. Shirer Nonlinear Hydrodynamic Modeling: A Mathematical Introduction, (Springer-Verlag, Heildelberg, 1987), pp. 180-230.

46. J.F. Drake, J.M. Finn, P. Guzdar, V. Shapiro, V. Shevchenko, A.B. Hassam, F. Waelbroeck, C.S. Liu, and R. Sagdeev, Peeling of convection cells and the generation of sheared flow, UMLPR Plasma Preprint, 1991.

47. E.N. Lorenz, Deterministic nonperiodic flow, J. Atmos. Sci. 20, 130-141 (1963).

48. A. McAllister, J. Mizushima, T. Tajima, and R.S Steinolfson, Vertical slot convection: a nonlinear simulation, in preparation, 1993.

49. A. McAllister and T. Tajima, Vertical slot convection: Nonlinear oscillation and chaos, in preparation, 1993.

50. H. Hanami and T. Tajima, Numerical study of compressible solar magnetoconvection with an open transitional boundary, Ap. J. 377, 694 (1991). 


\section{Figure Captions}

1. The Vertical Slot Convection Problem. The hot wall is at $T_{2}$ and the cold wall at $T_{1}$, separated by a distance $2 L$. The arrow labeled " $g$ " indicates the gravitational acceleration.

2. The real part $(\lambda)$ of the VSC eigenvalues plotted against the Grashof number. a) shows $\operatorname{Pr}=7.5$ and $\alpha=1.383$, with $\lambda$ ranging from -0.25 to 0.033 , and $G$ from 50 to 750 . b) shows $\operatorname{Pr}=50.0$ and $\alpha=0.9$, with $\lambda$ ranging from -0.125 to 0.017 , and $G$ from 10 to 750 . In both cases the horizontal line indicated by the arrow is $\lambda=0$.

3. The VSC neutral stability curve, $\lambda(G, \alpha)=0$, at $P r=7.5$. $G$ ranges from 0 to 10,000 and $\alpha$ from 0.0 to 5.0 .

4. The critical Grashof and wavenumbers for the VSC stationary mode, plotted against the log of Prandtl number. The $G$ scale is from 455 to 511 , the o scale from 1.337 to 1.411, for $\operatorname{Pr}$ from 0.001 to $\sim 12$.

5. Contour maps of $\lambda_{\max }(G, \alpha)$ for the VSC problem at Prandtl numbers $0.001,0.25,0.5$, and 15. $G$ ranges from 1.0 to 10,000 and $\alpha$ from 0.0 to 10.0. The contours are from $-0.10,-0.056$ in the last frame, to 0.016 at intervals of 0.004 .

6. Contour maps of $\gamma_{\max }(G, \alpha)$ for the VSC problem at Prandtl numbers $0.001,0.25,0.5$, and 7.5. $G$ ranges from 1.0 to 10,000 and $\alpha$ from 0.0 to 10.0. The contours are from 0.0 to $0.58,0.62$ in the last frame, at intervals of 0.02 .

7. Contour maps of $\lambda_{\max }(G, \alpha)$ for the VSC problem at Prandtl numbers $12.5,15$, and 100. a) $G$ ranges from 50 to 1,000 and $\alpha$ from 0.0 to 2.0. The contours are from -0.018 to 0.0099 at intervals of 0.0009 . b) $G$ ranges from 100 to 5,000 and $\alpha$ from 0.0 to 5.0. 
The contours are from -0.036 to 0.003 at intervals of 0.001 . c) $G$ ranges from 20 to 1,000 and $\alpha$ from 0.0 to 3.0. The coritours are from -0.0144 to 0.0096 at intervals of 0.0008 .

8. The real part $(\lambda)$ of the Benard eigenvalues plotted against the Grashof number. The case shown is for $\operatorname{Pr}=7.5$ and $\alpha=1.5585$. $\lambda$ ranges from -0.05 to 0.037 , and $G$ from 1 to 500 . The horizontal line indicated by the arrow is $\lambda=0$.

9. Contour maps of $\lambda_{\max }(G, \alpha)$ for the Benard problem at $\operatorname{Pr}=10 . G$ ranges from 1.0 to 100 and $\alpha$ from 0.0 to 10.0 . The contours are from -0.1 to 0.020 at intervals of 0.004 .

10. The critical Grashof and wavenumbers for the Benard-with-Shearflow problem, plotted against the $\log$ of Prandtl number. The $G$ scale is from 277 to 514 , the $\alpha$ scale from 1.337 to 1.411 , for $\operatorname{Pr}$ from 0.001 to 10 .

11. Contour maps of $\lambda_{\max }(G, \alpha)$ and $\gamma_{\max }(G, \alpha)$ for the Bei.ard-with-Shearflow problem at $P r=0.001$ and 15.0. $G$ ranges from 1.0 to $10,000(\mathrm{a} \& \mathrm{c})$ and $100(\mathrm{~b} \mathrm{\&} \mathrm{d})$ ard $\alpha$ from 0.0 to 10.0. a) The contours are from -0.10 to 0.016 at intervals $n f 0.004$. b) The contours are from -0.03 to 0.008 at intervals of 0.001 . c) The contours are from 0.0 to 0.590 at intervals of 0.2 . d) The contours are from 0.0 to 0.309 at intervals of 0.1 .

12. Contour maps of $\lambda_{\max }(G, \alpha)$ for the VSC zero-state problem, no initial flow and zero temperature, at $\operatorname{Pr}=0.75$. $G$ ranges from 1.0 to 10,000 and $\alpha$ from 0.0 to 10.0 . The contours are from -0.012 to 0.004 at intervals of 0.004 .

13. A complex pair of stream function eigenmodes. These are for $G=1000, \operatorname{Pr}=7.5$, and $\alpha=8.0$.

14. A typical near critical Benard eigenmode; $\hat{\psi}, \widehat{\omega}$, and $\hat{T} . G=14.23, \operatorname{Pr}=7.5, \alpha=$ $1.558, \lambda=-0.408 \times 10^{-5}$, and $\gamma=0.0$. 
15. The Benard low $\operatorname{Pr}$ overall phase variation plotted as a function of $G$ and $\alpha . G$ ranges from 1.0 to 10,000 and $\alpha$ from 0.0 to 10.0 . The mottled region has a phase of 0 , the lower left one of $\frac{2}{\pi}$ and the lower right one of $-\frac{2}{\pi}$.

16. A sample Zero-state mode. $G=1000.0, \operatorname{Pr}=7.5, \alpha=4.0, \lambda=0.725 \times 10^{-2}$, and $\vartheta=0.0$.

17. The VSC zero-state case high $\operatorname{Pr}$ overall phase variation plotted as a function of $G$ and $\alpha . G$ ranges from 1.0 to 10,000 and $\alpha$ from 0.0 to 10.0 . The mottled region has a phase of 0 , and the blank area one of $-\frac{2}{\pi}$.

18. The overall phases of the VSC eigenmodes. $G$ ranges from 1.0 to 10,000 and $\alpha$ from 0.0 to 10.0 . The main phases are: $-\frac{2}{\pi}$, horizontal stripes; 0 , vertical stripes; $\frac{2}{\pi}$, positive definite siripes; and $\pi$, negative definite stripes. Regions of other phases are shown with overlaid stripes indicating the quadrant those phases lie in. The background is a contolir map of these phases.

19. The internal phases of the VSC eigenmodes. $G$ ranges from 1.0 to 10,000 and $\alpha$ from 0.0 to 10.0. Those modes which when "lipped" (see the text) have out of phase temperature eigenfields are shown in negative definite stripes, those with out of phase kinetic eigenfields in vertical stripes. Half wavelength modes are in horizontal stripes,and the "in-phase" modes in positive definite stripes. The background is a contour map of the overall phases.

20. The mode property regimes plotted against the Prandtl number $(0.001$ to $\sim 12)$, with $G_{c}$ and $\alpha_{c}$ shown for reference. The horizontal lines indicate a the property ranges, the vertical lines mark specific positions.

21. a) A low $\operatorname{Pr}$, real VSC mode. $G=2000.0, \operatorname{Pr}=0.001, \alpha=1.5, \lambda=0.137 \times 10^{-1}$, and $\gamma=0.0$. b) A low Pr. complex VSC mode. $G=1500.0, \operatorname{Pr}=0.001, \alpha=2.5$, 
$\lambda=-0.641 \times 10^{-1}$, and $\gamma=0.618 \times 10^{-1}$. c) The arrowhead mode, at high $G$, high $\alpha . \quad G:=9000.0, \operatorname{Pr}=0.001, \alpha=9.0, \lambda=-0.323 \times 10^{-1}$, and $\gamma=0.524$. d) A strong reverse pitch mode. $G=2500.0, \operatorname{Pr}=0.001, \alpha=2.5, \lambda=-0.500 \times 10^{-1}$, and $\gamma=0.111$.

22. a) The thermal half wavelength mode. $G=5.000, \operatorname{Pr}=0.1, \alpha=2.7, \lambda=-0.391 \times 10^{-1}$, and $\gamma=0.125$. b) The VSC linear critical mode at $\operatorname{Pr}=7.5 . G=491.820, \operatorname{Pr}=7.5$, $\alpha=1.383, \lambda=0.481 \times 10^{-7}$, and $\gamma=0.0$. c) A typical high $\alpha$ high $\operatorname{Pr}$ complex mode. $G=1500.0, \operatorname{Pr}=7.5, \alpha=3.5, \lambda=-0.790 \times 10^{-2}$, and $\gamma=0.218$. d) The complex critical modes. $G=492.060, \operatorname{Pr}=12.450, \alpha=0.342, \lambda=-0.391 \times 10^{-6}$, and $\gamma=0.204 \times 10^{-1}$. 


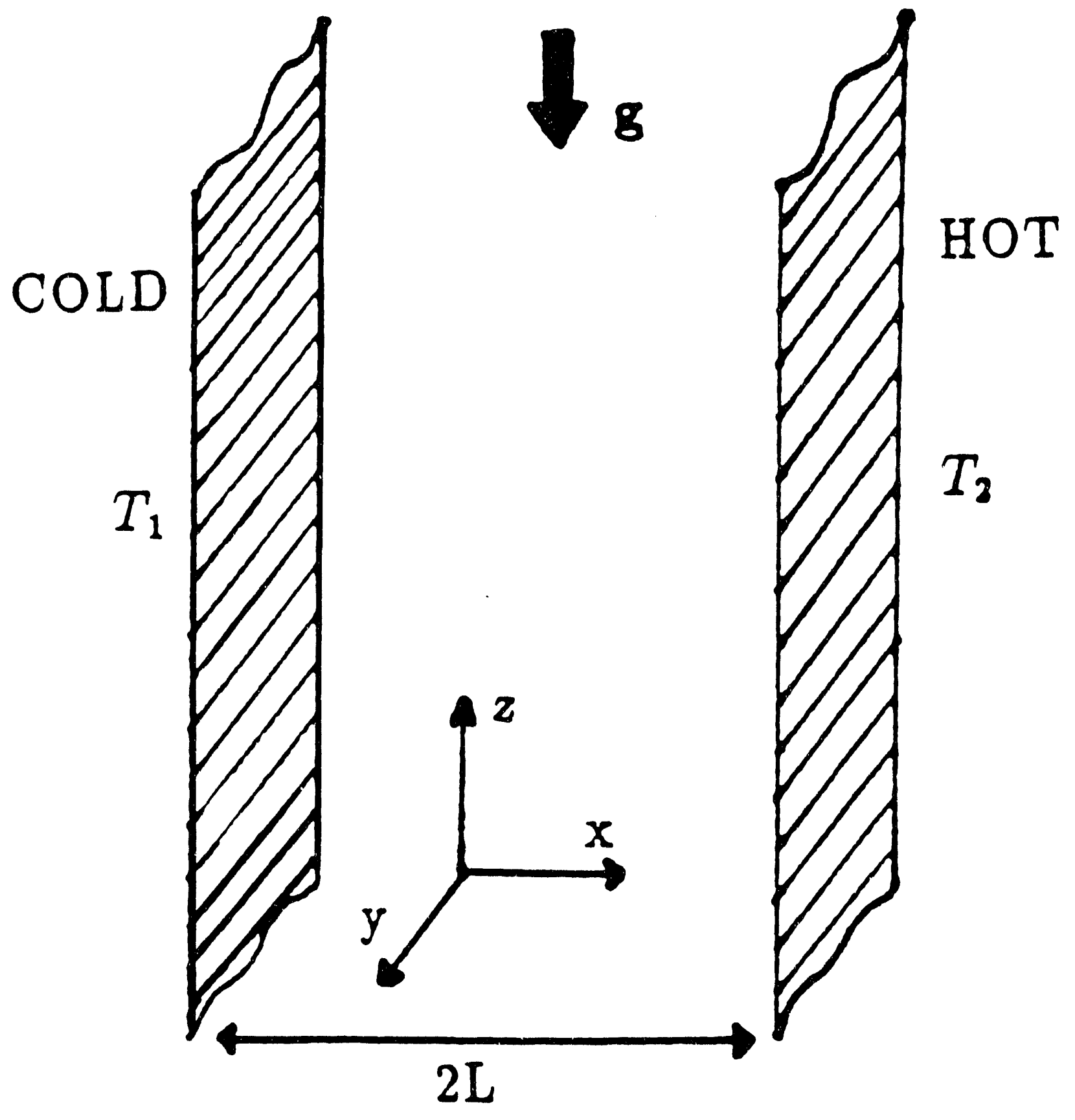

Fig. 1 

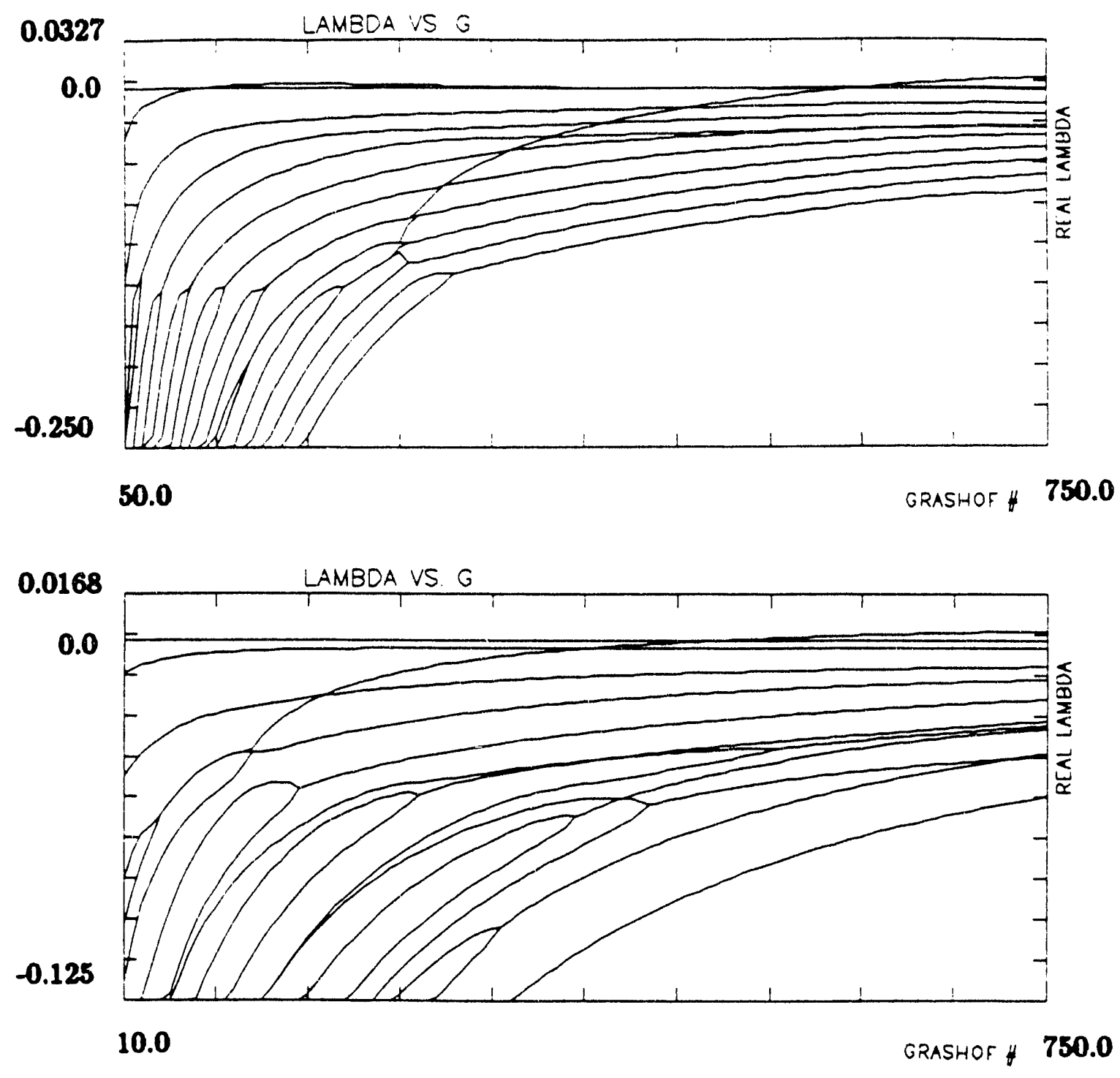

Fig. ? 


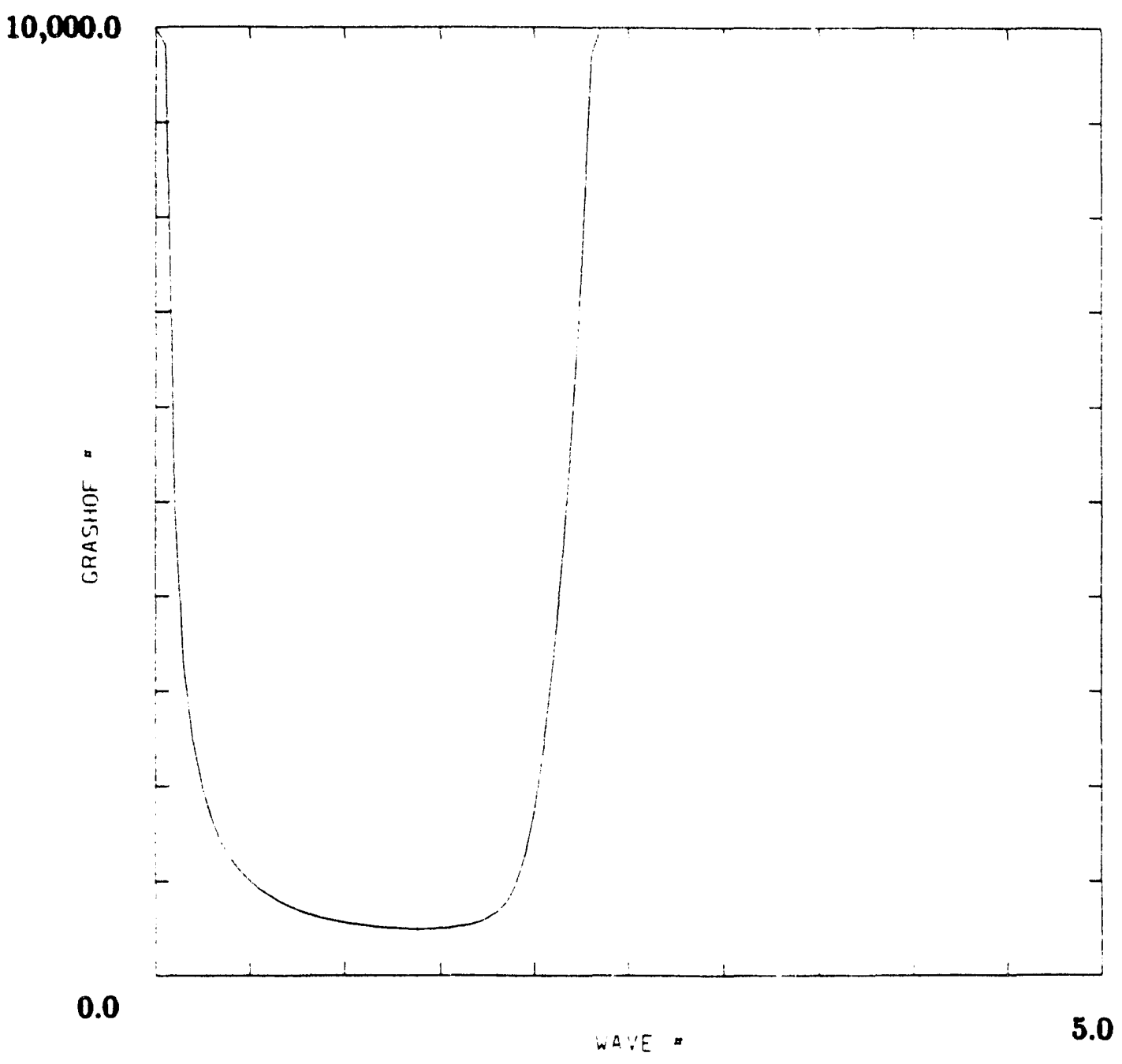

Fig. 3 


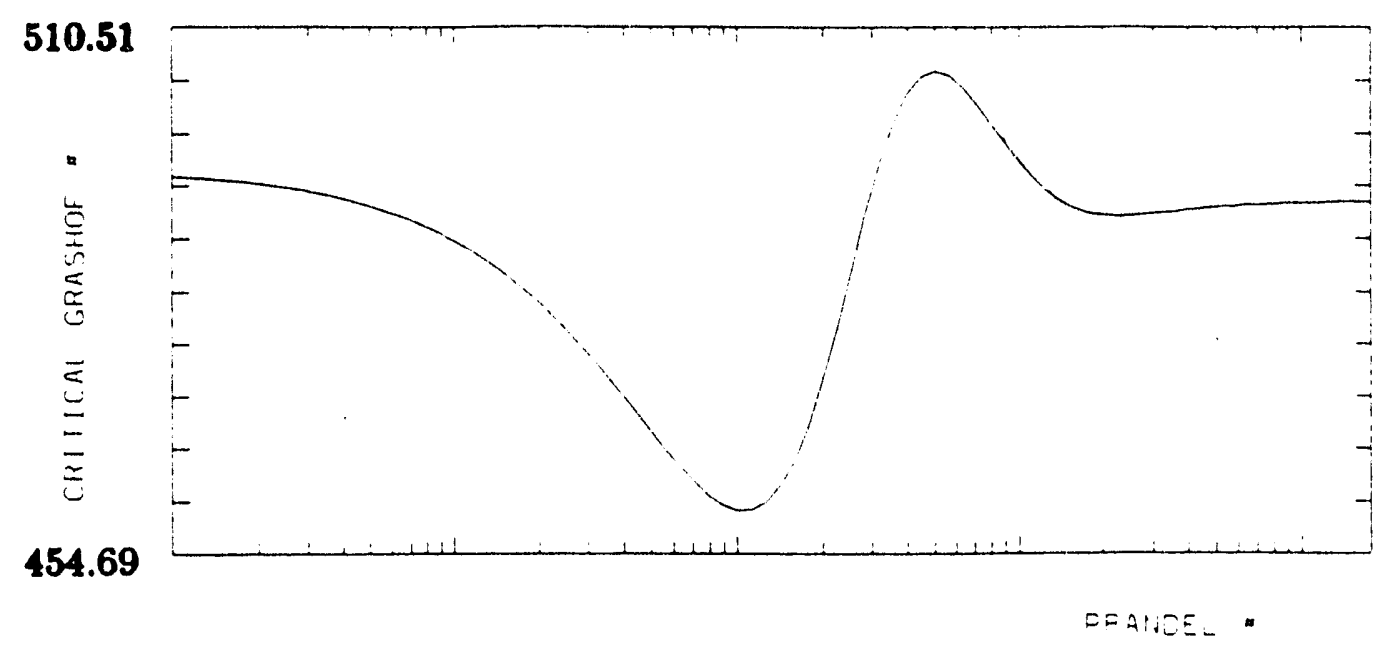

(a)

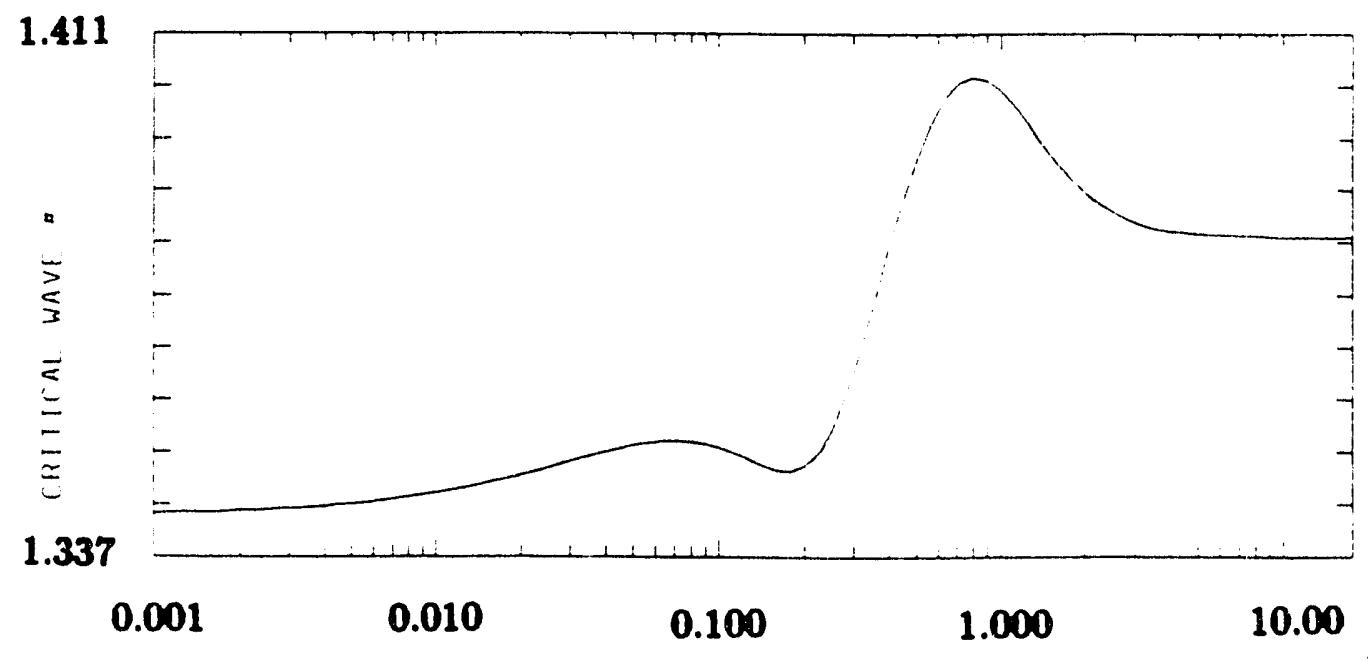

(b)

Fig. 4 


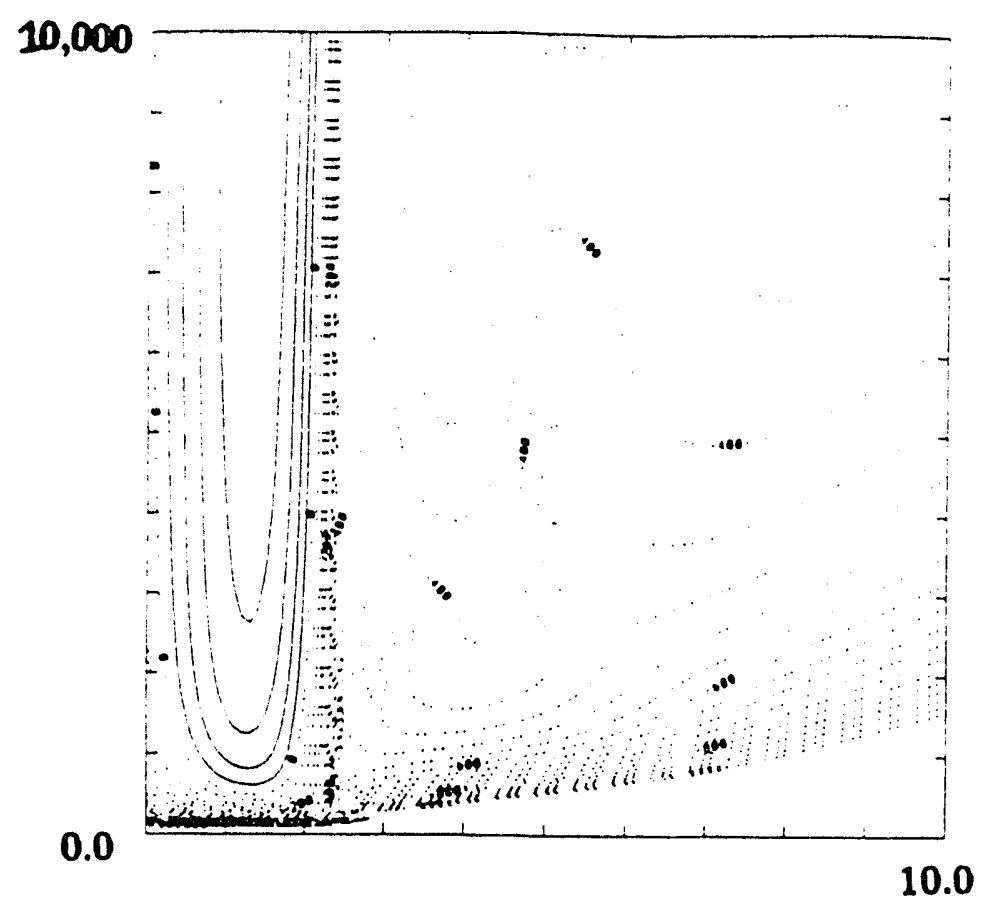

(a)

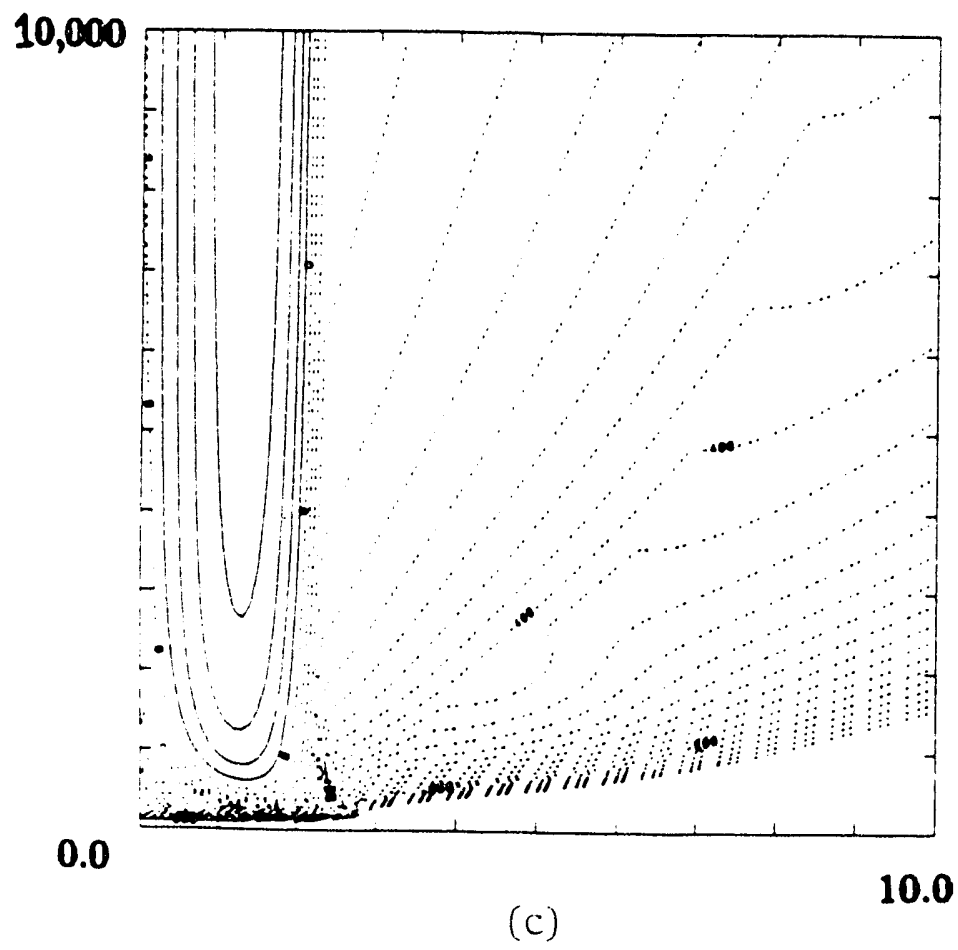

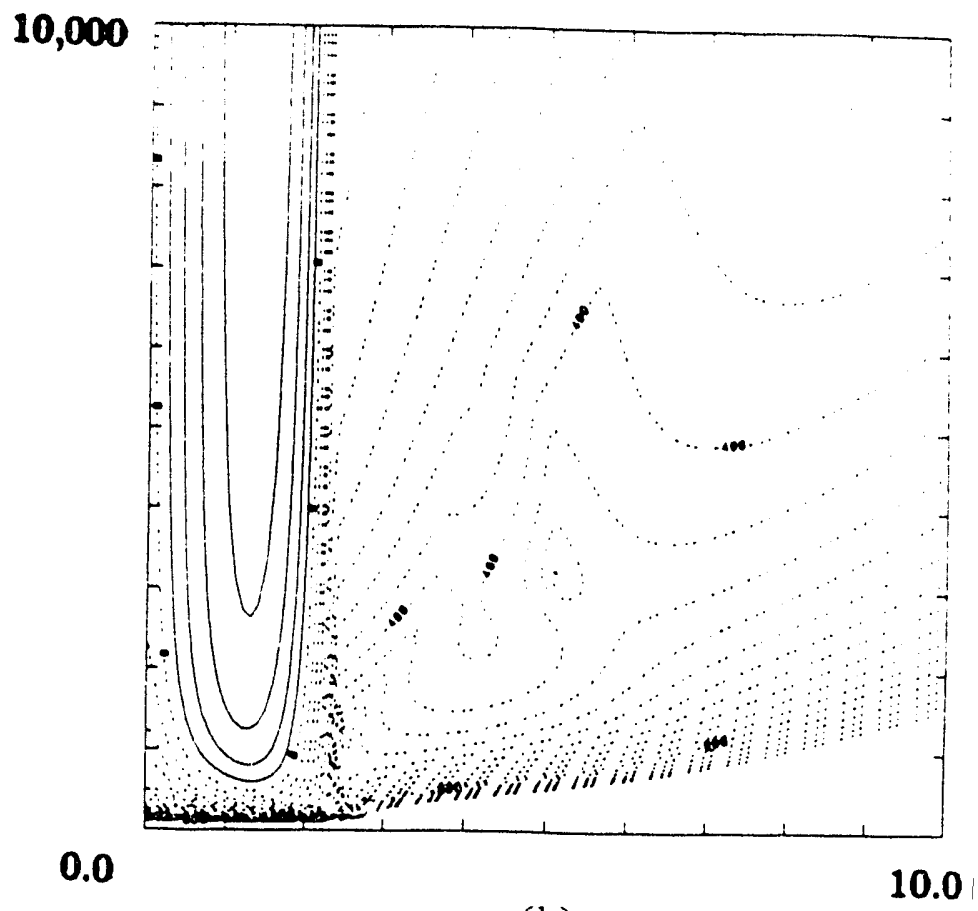

(b)

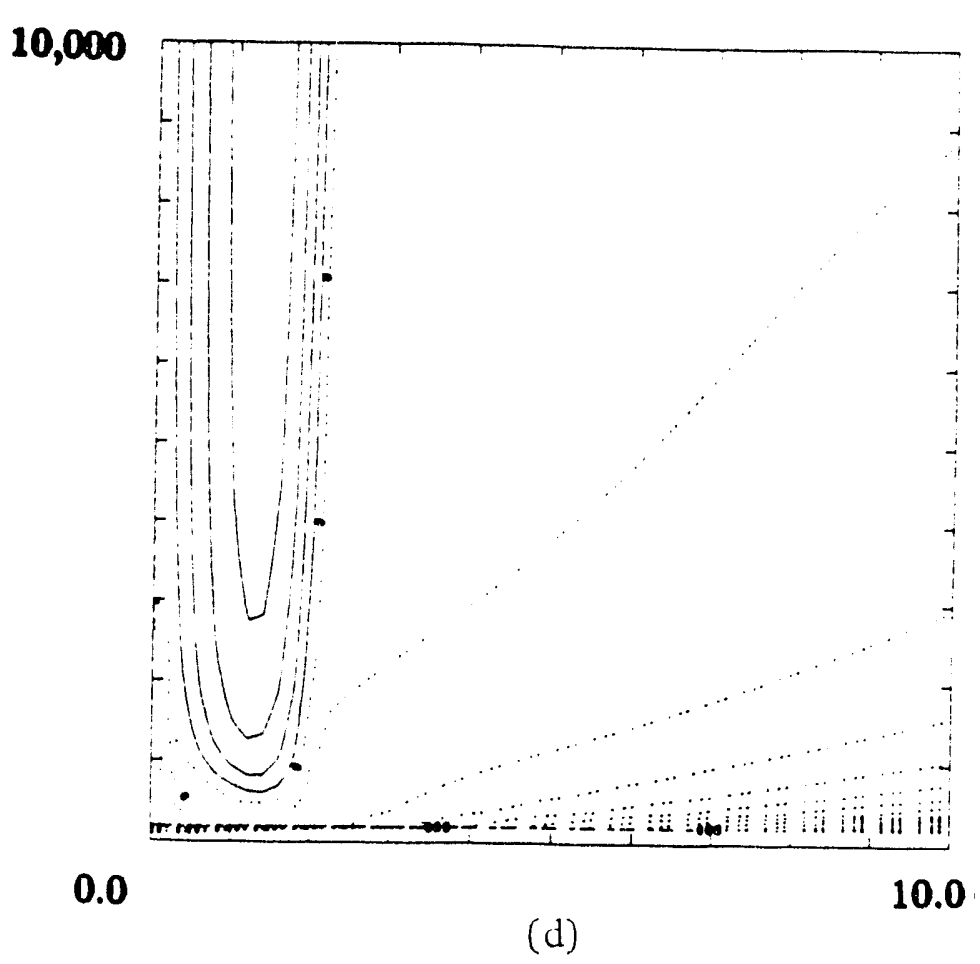

Fig. 5 


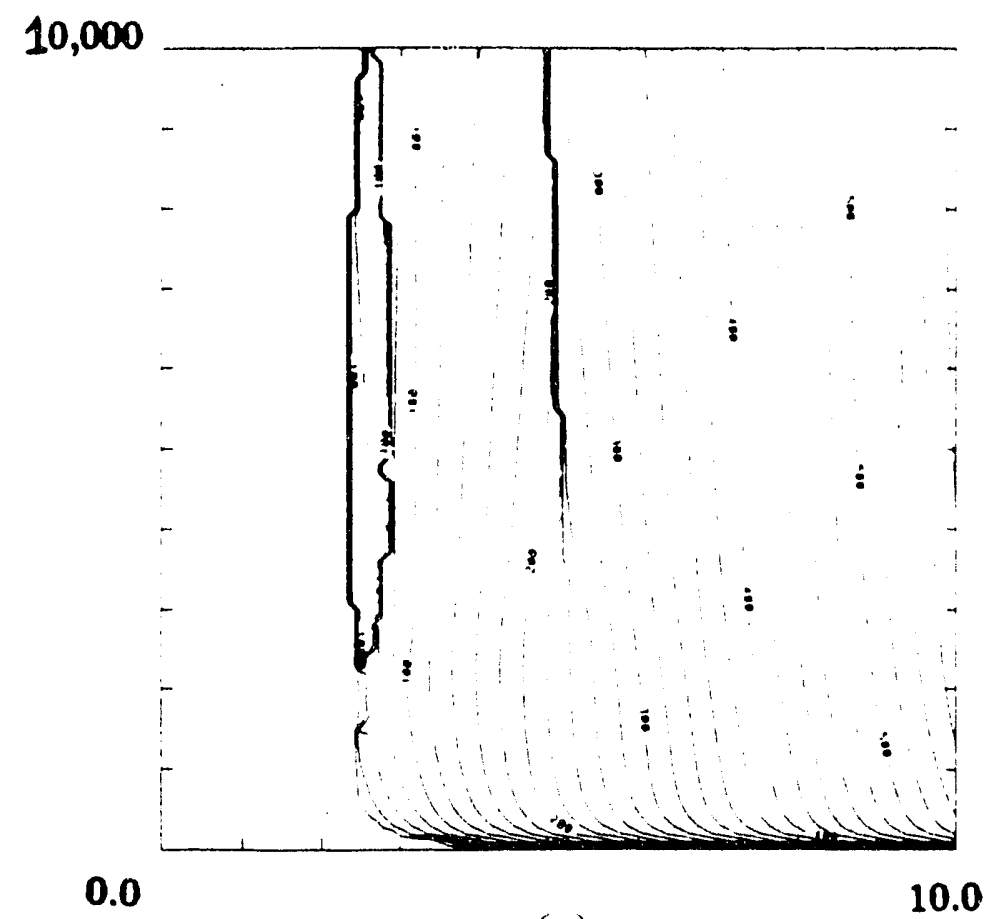

(a)

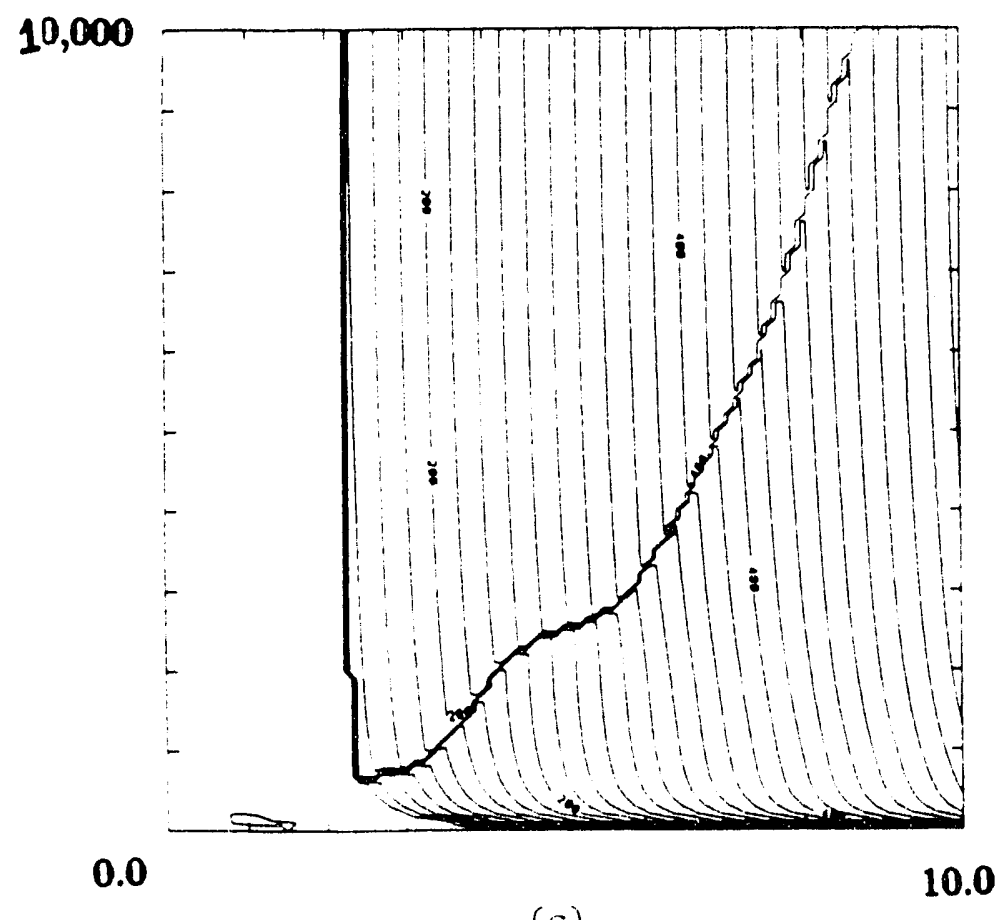

(c)
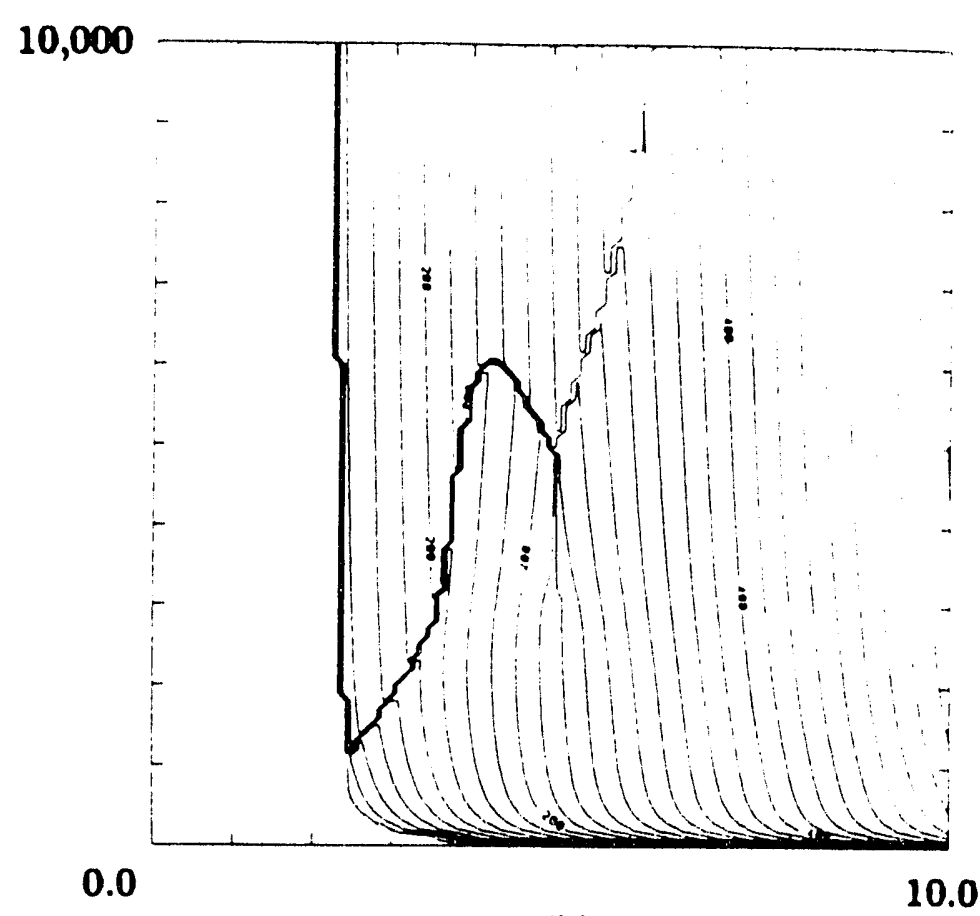

(b)

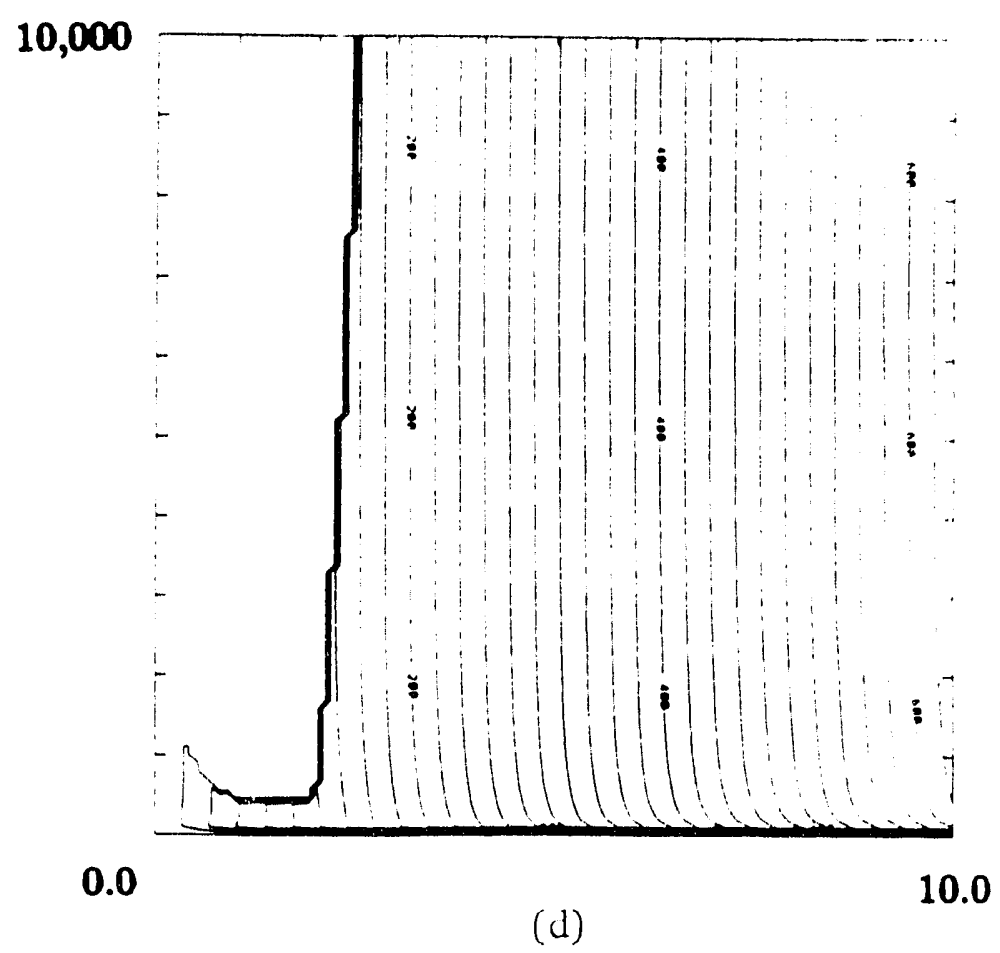

Fig. 6 

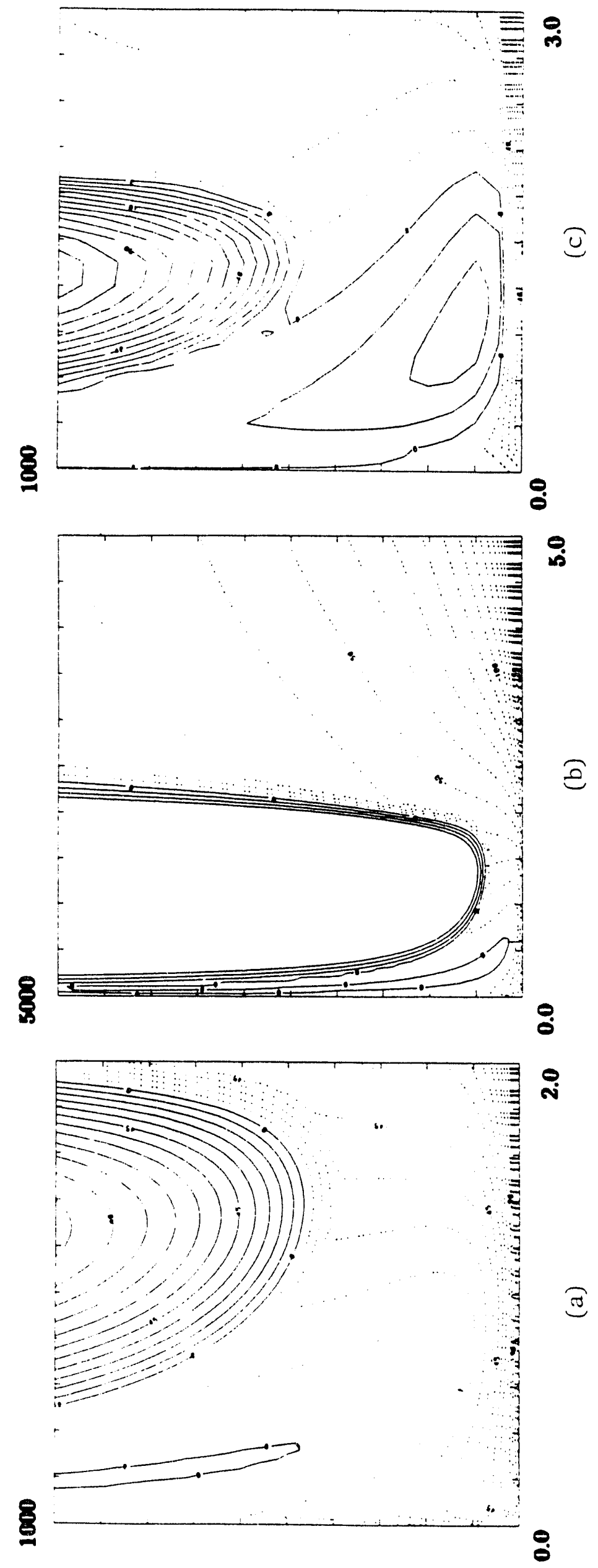

Fig. 7 


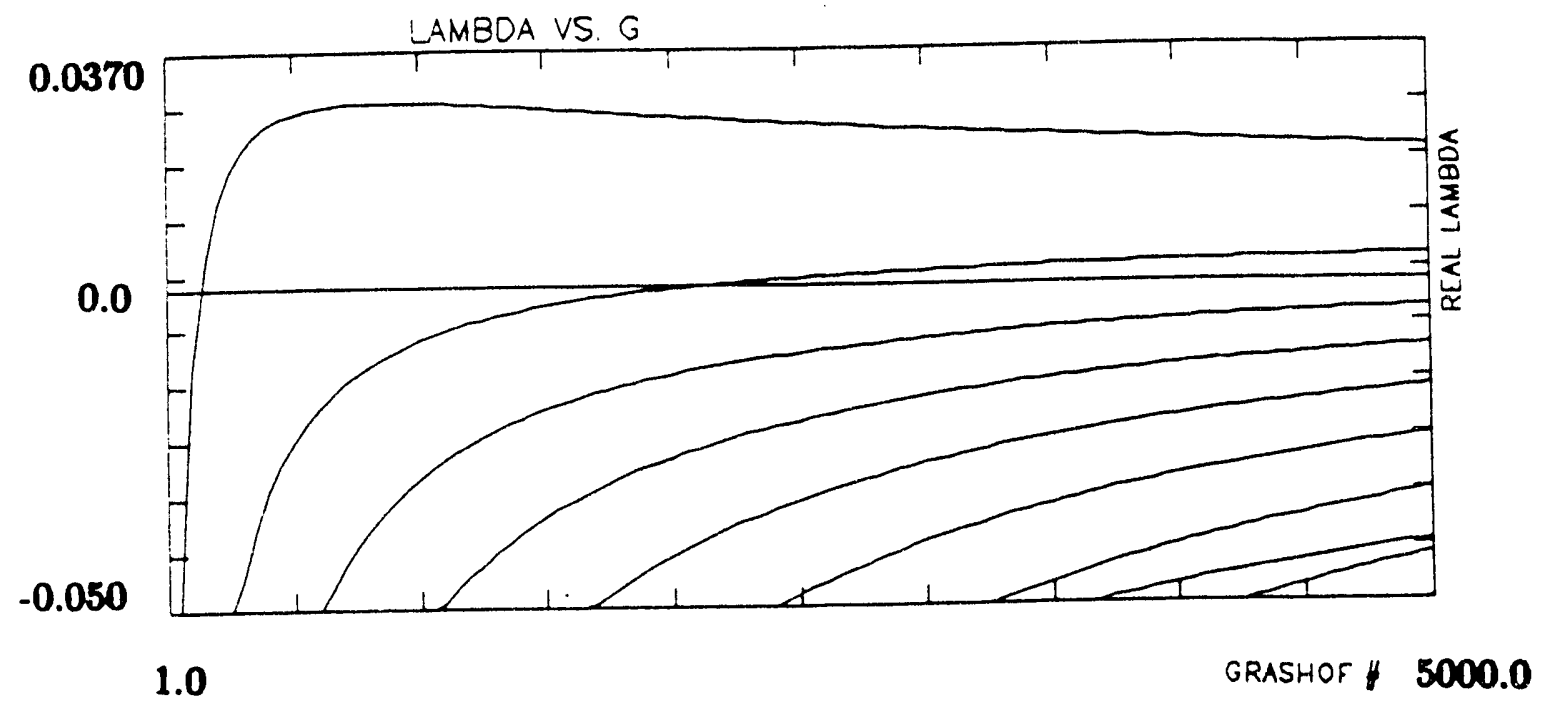

Fig. 8 


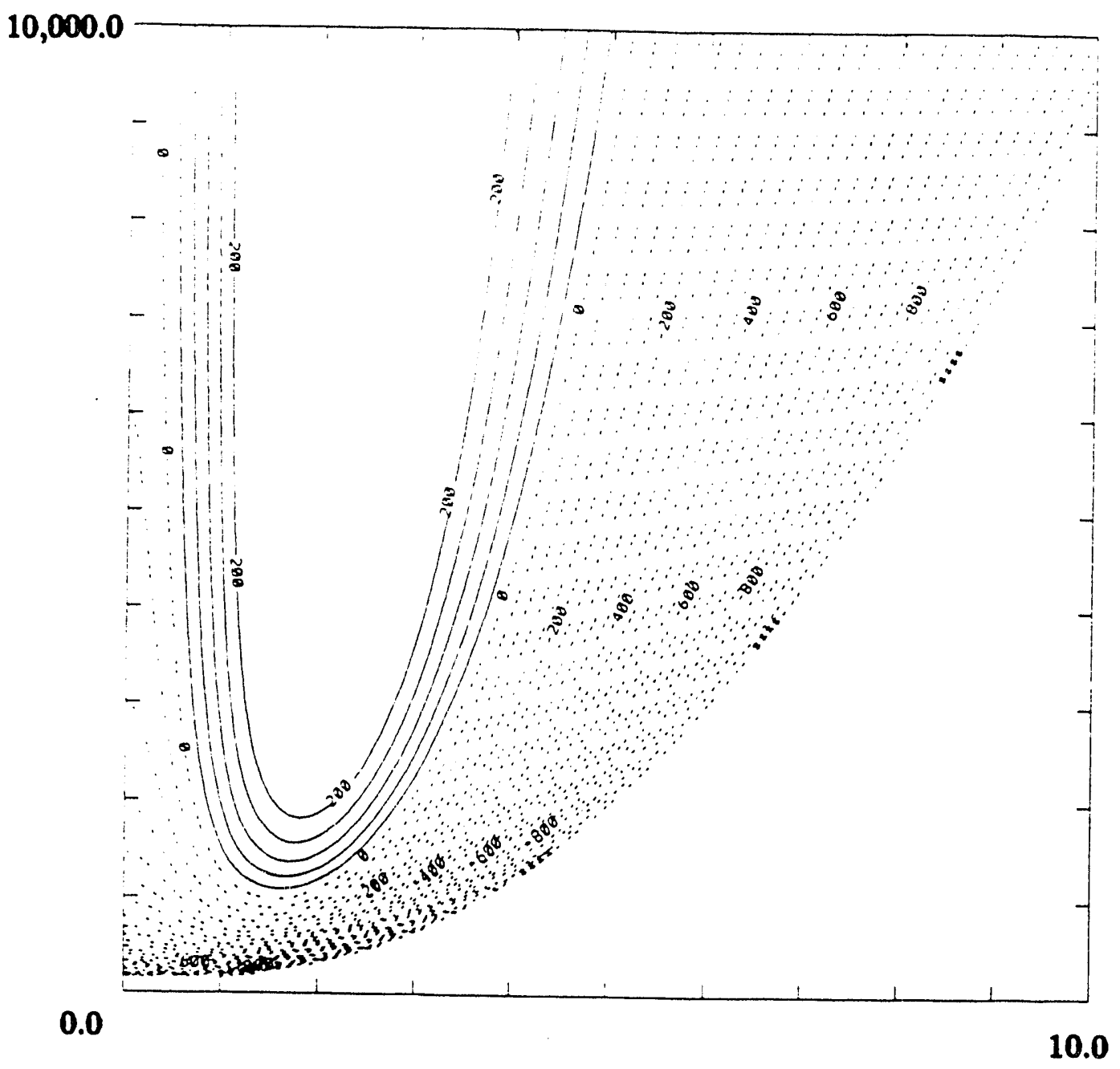

Fig. 9 


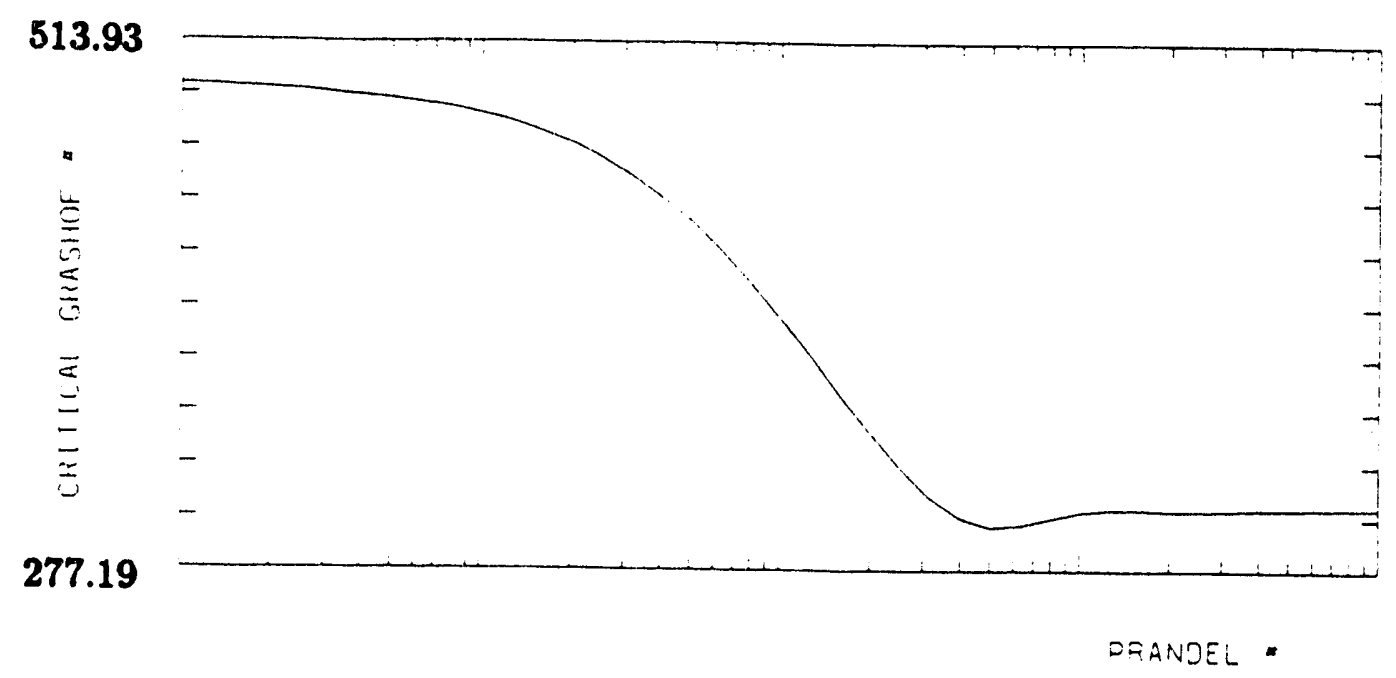

(a)

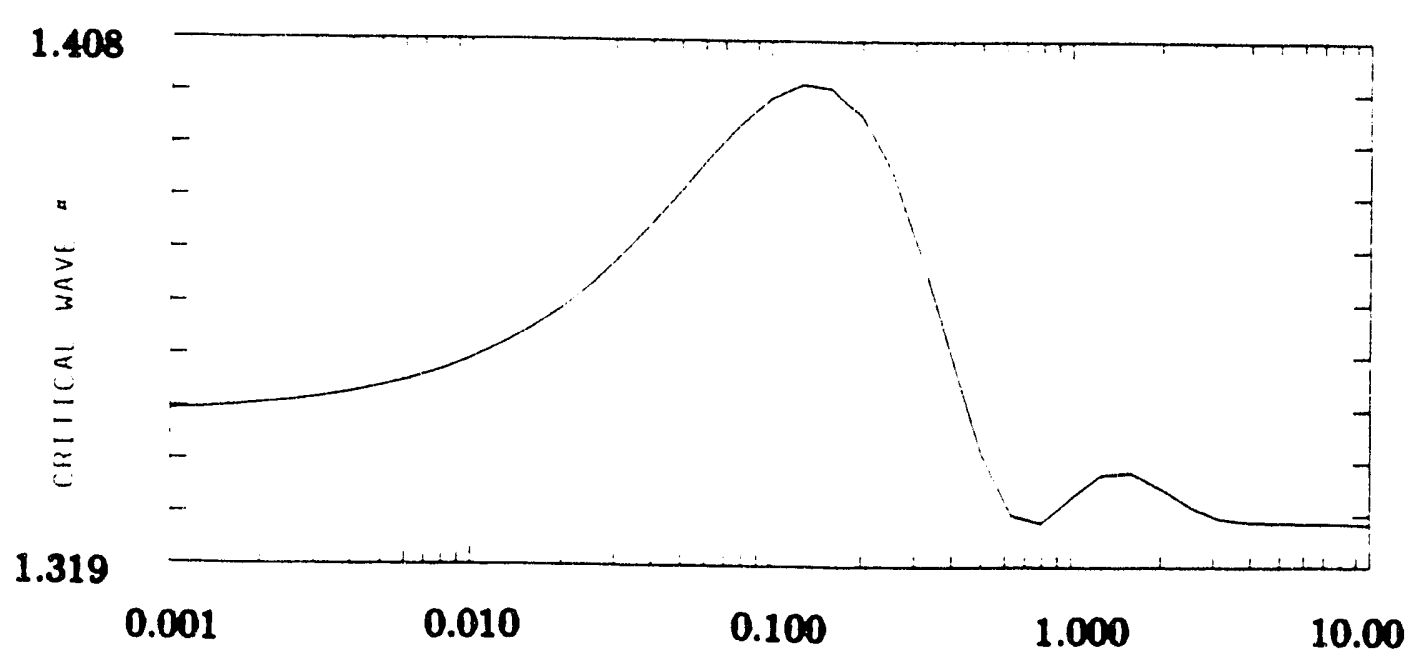

(b)

Fig. 10 


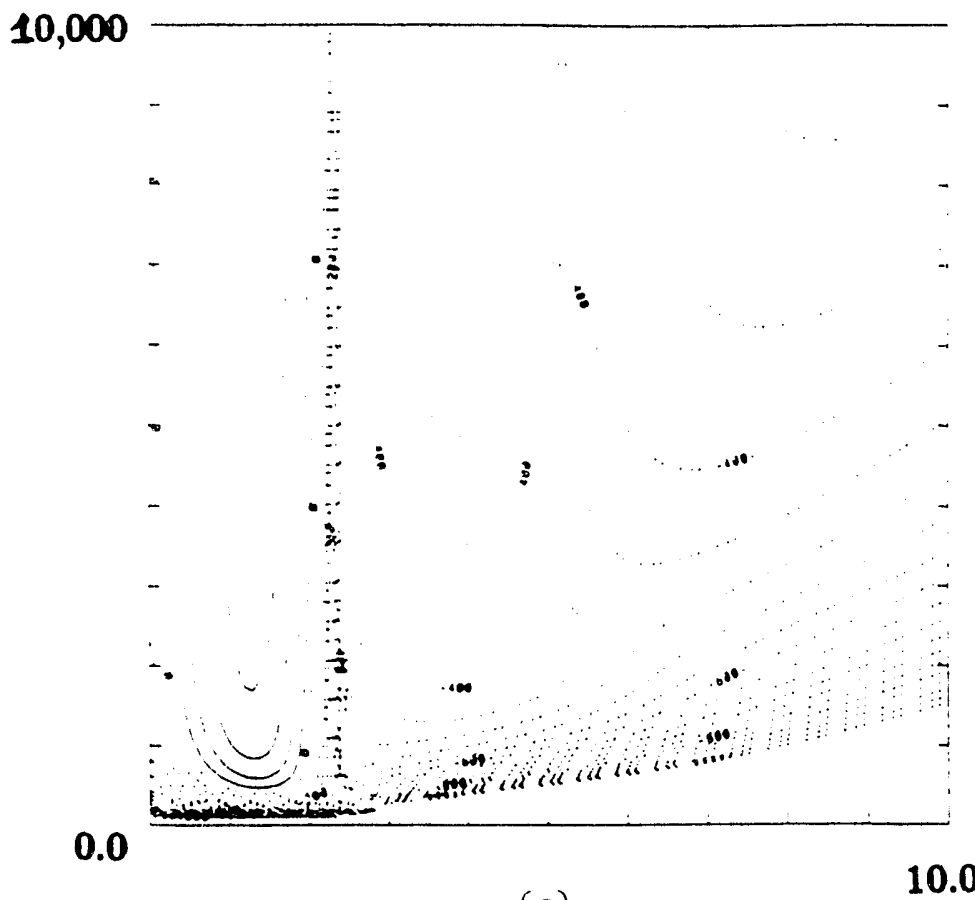

(a)
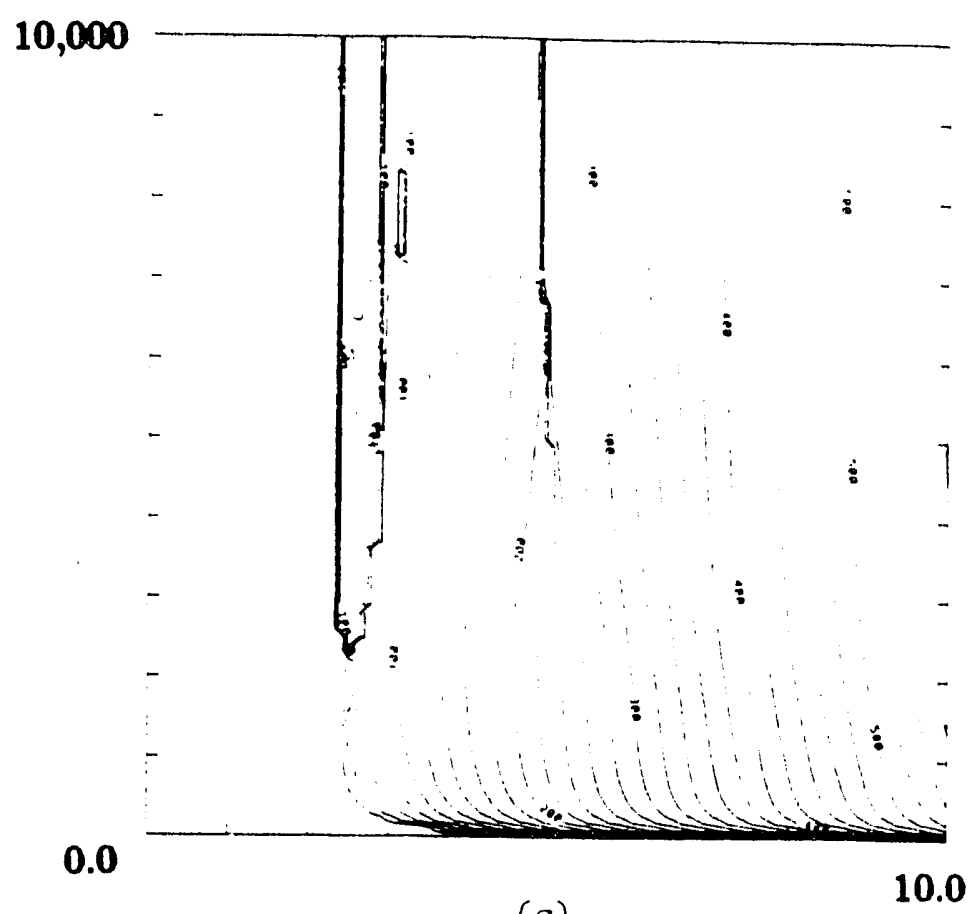

(c)

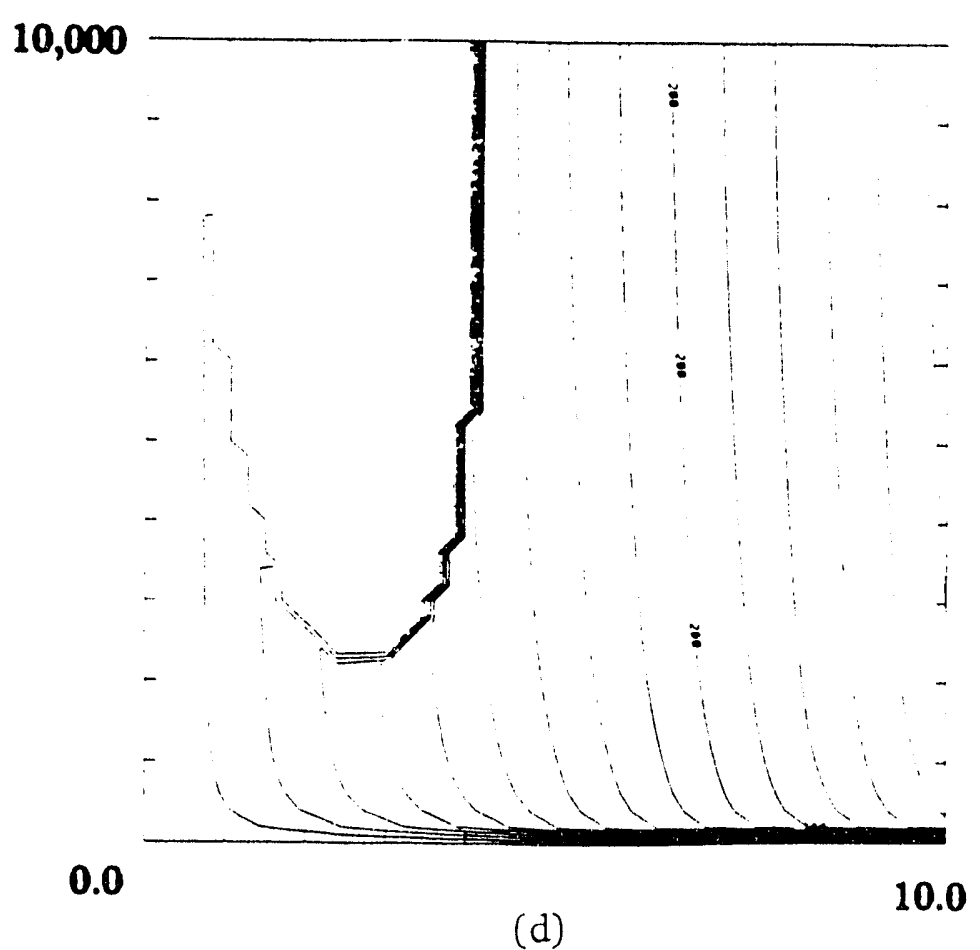

10,000

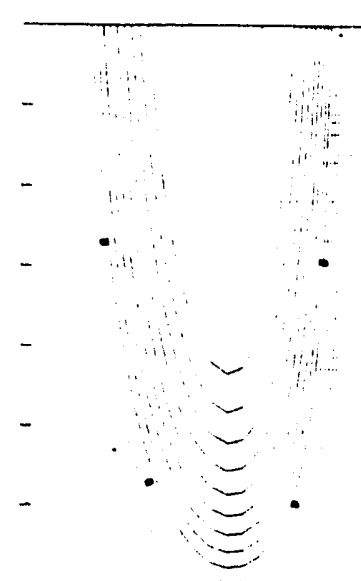

n. 10.0

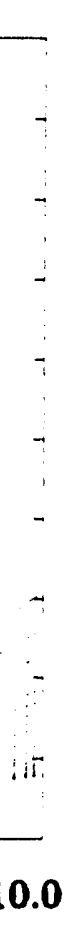

(b)

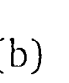




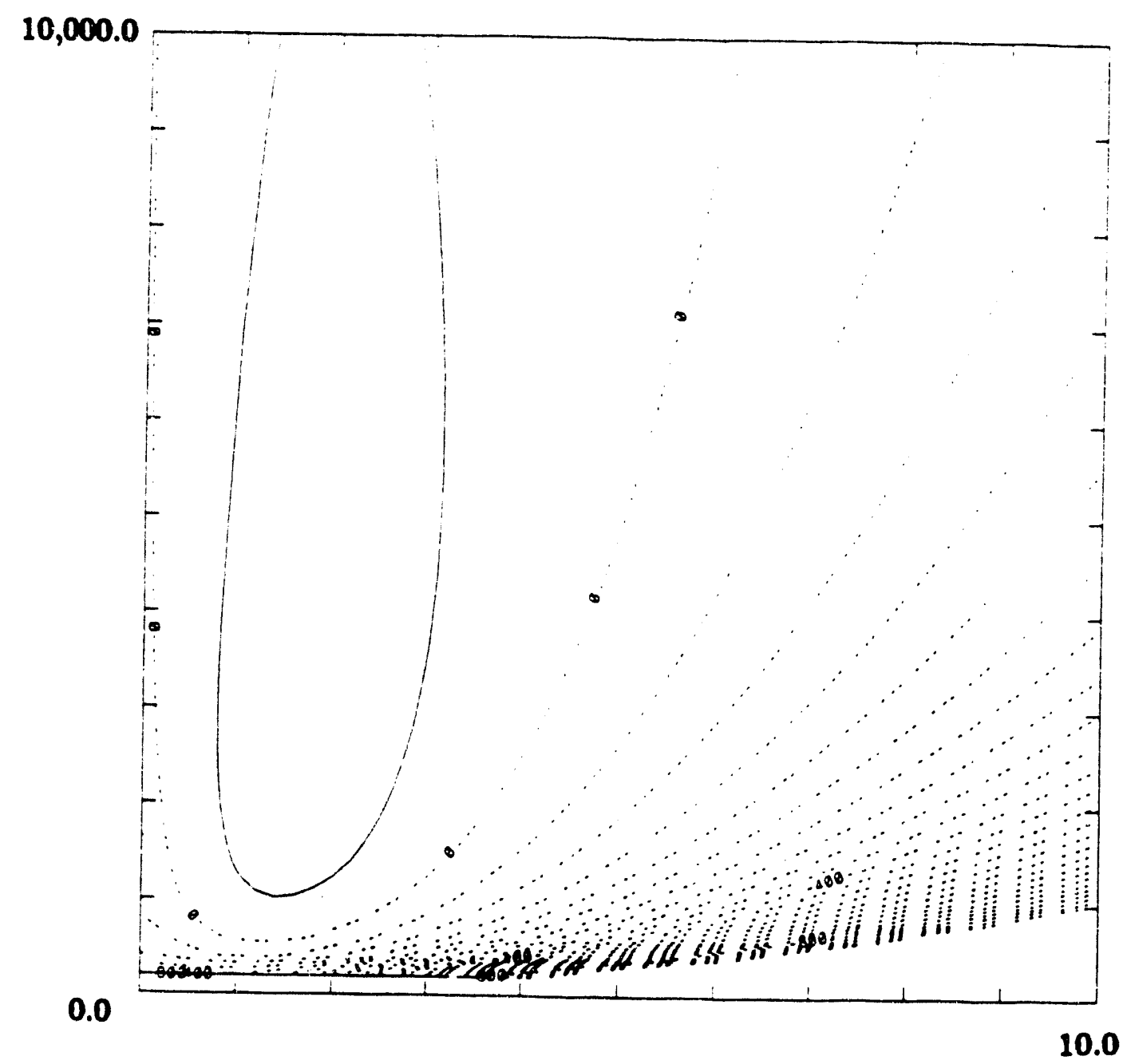

Fig. 12 

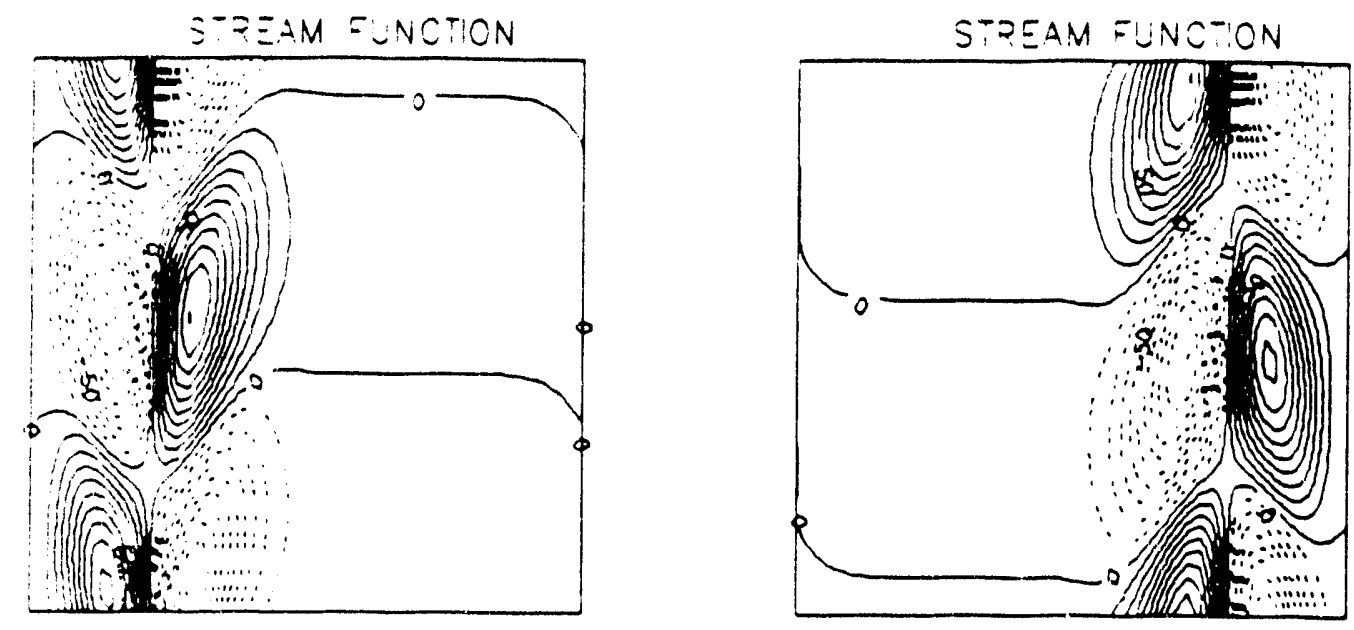

Fig. 13 

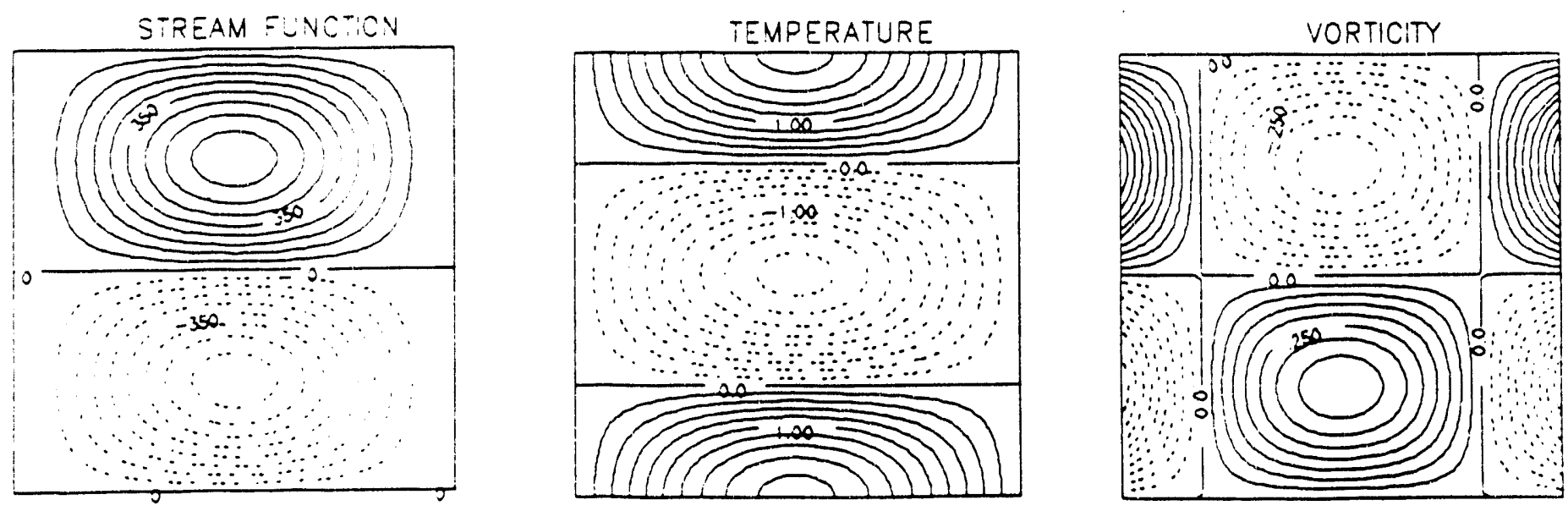

Fig. 14 


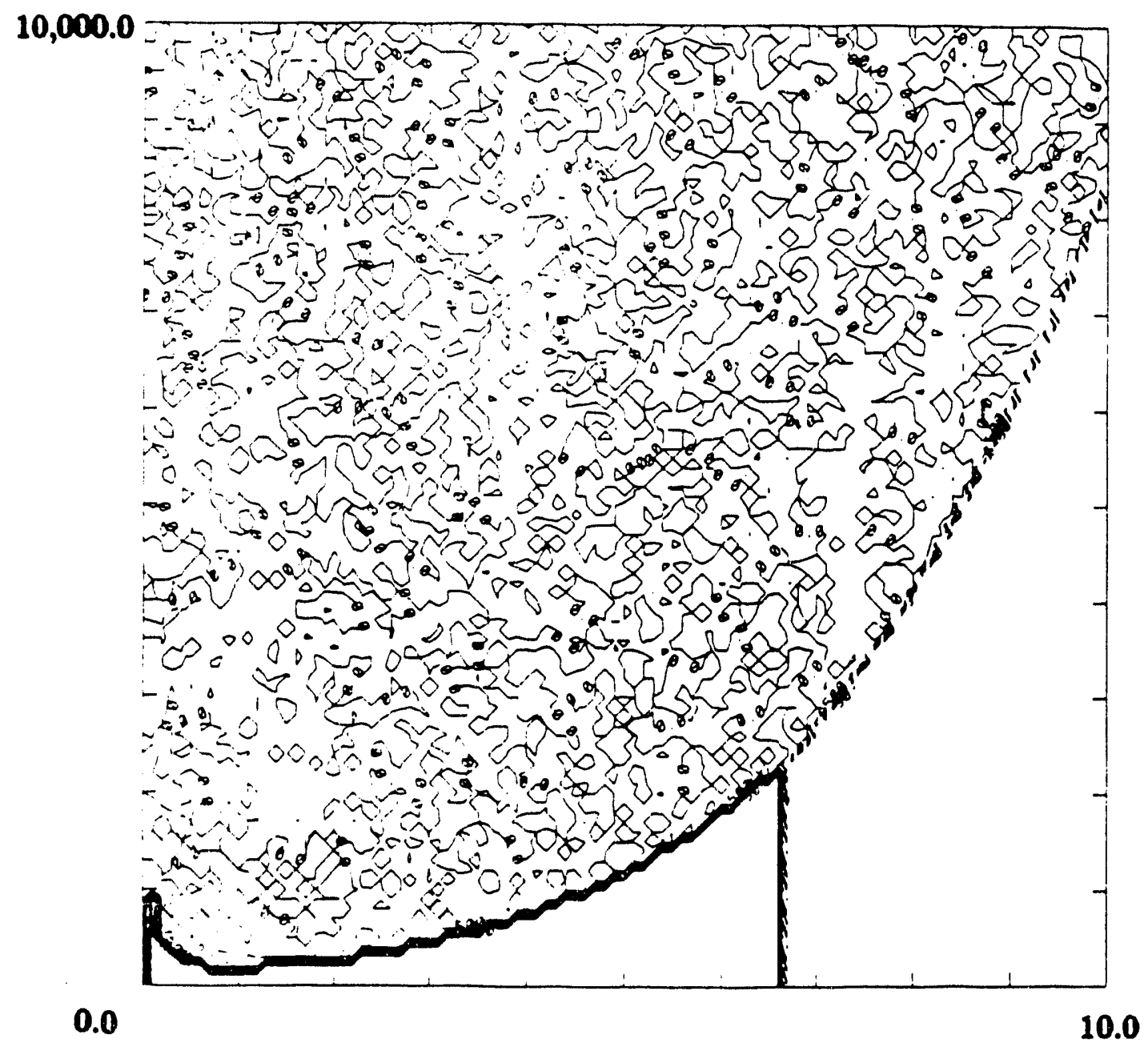

Fig. 15 

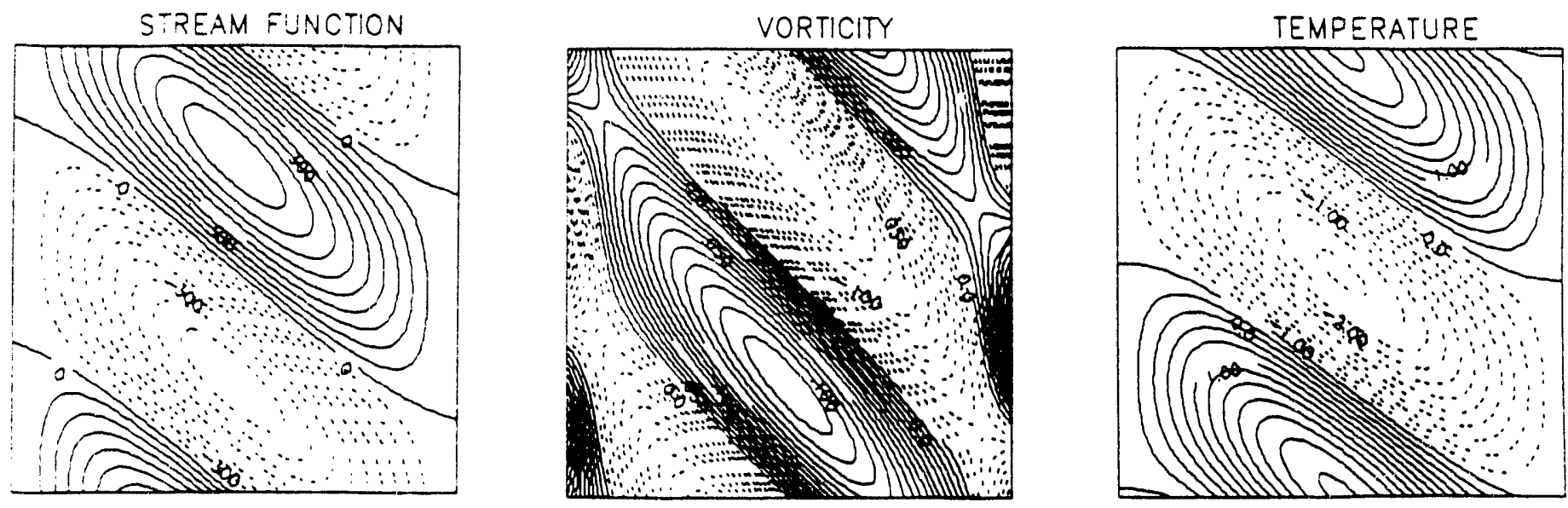

Fig. 16 


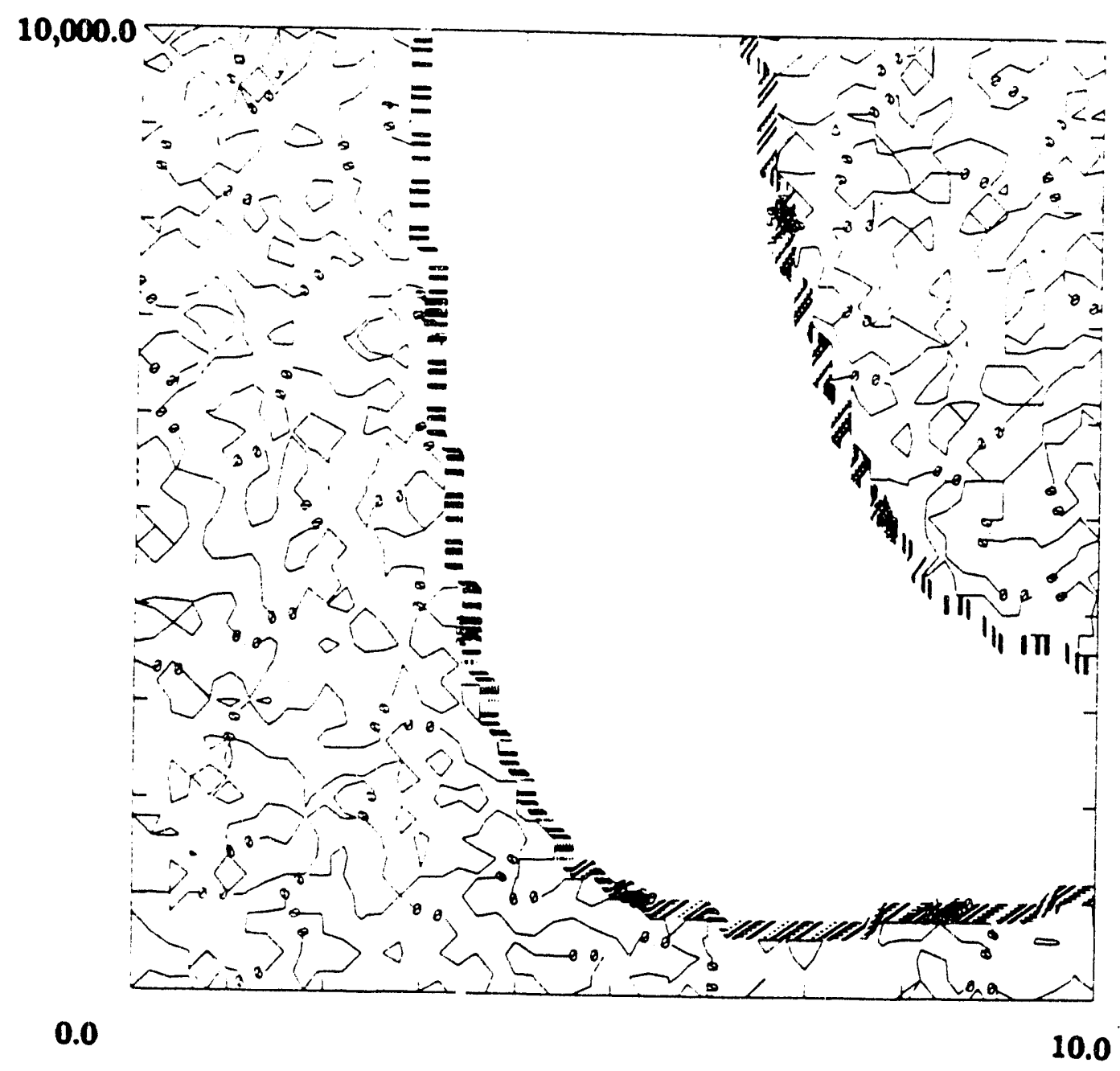

Fis. 17 

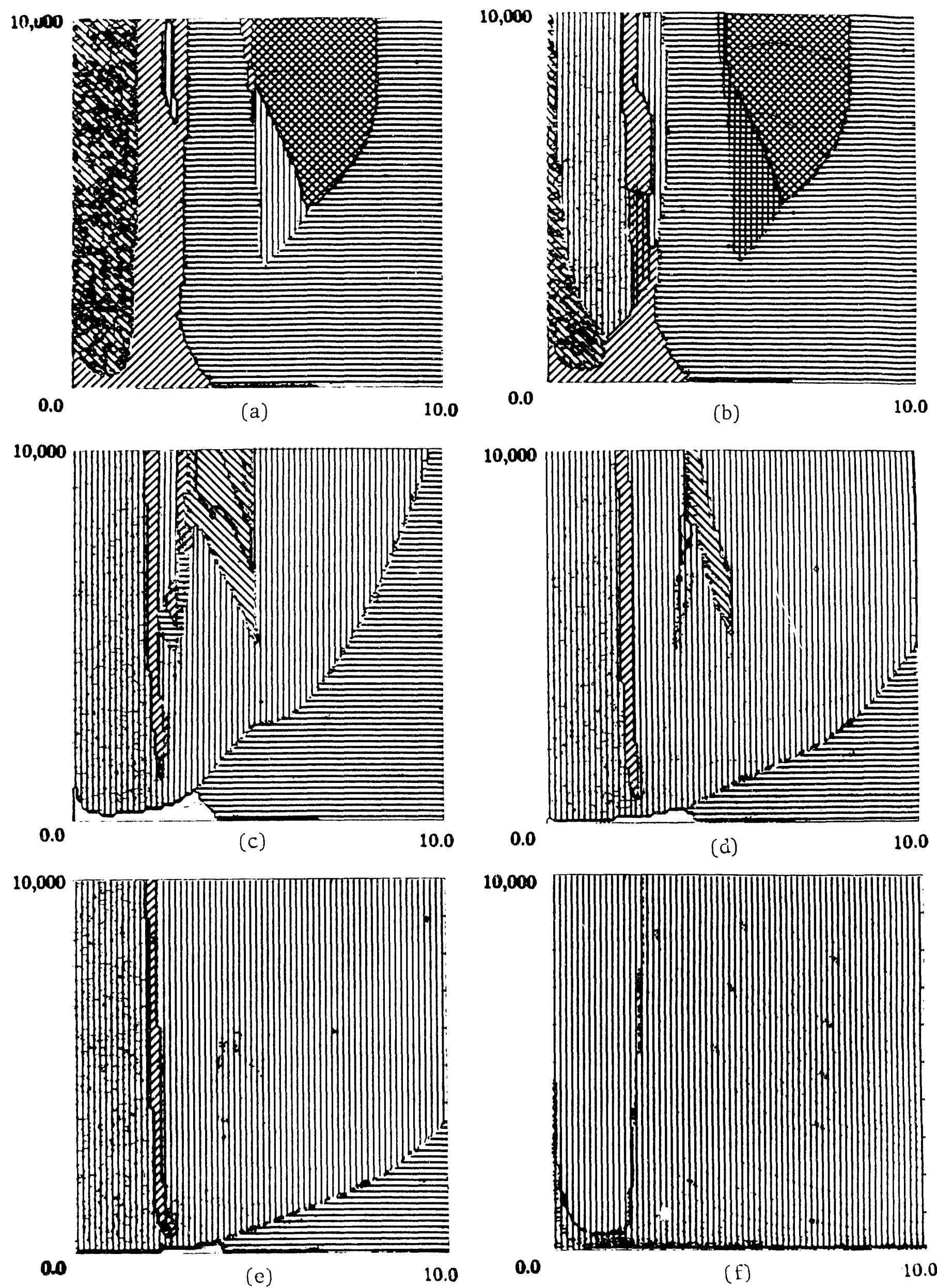

(f)

10.0 

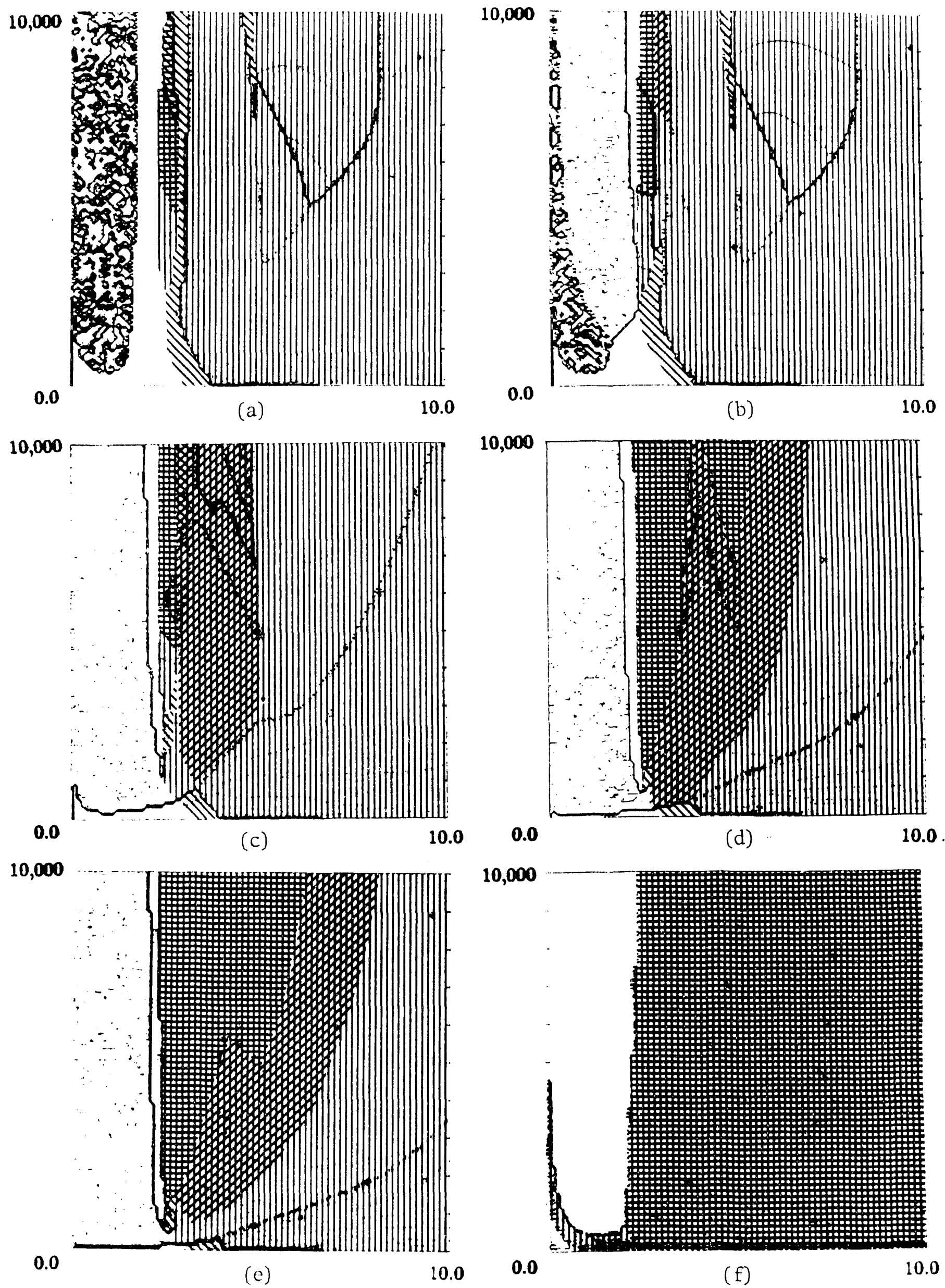

10,000
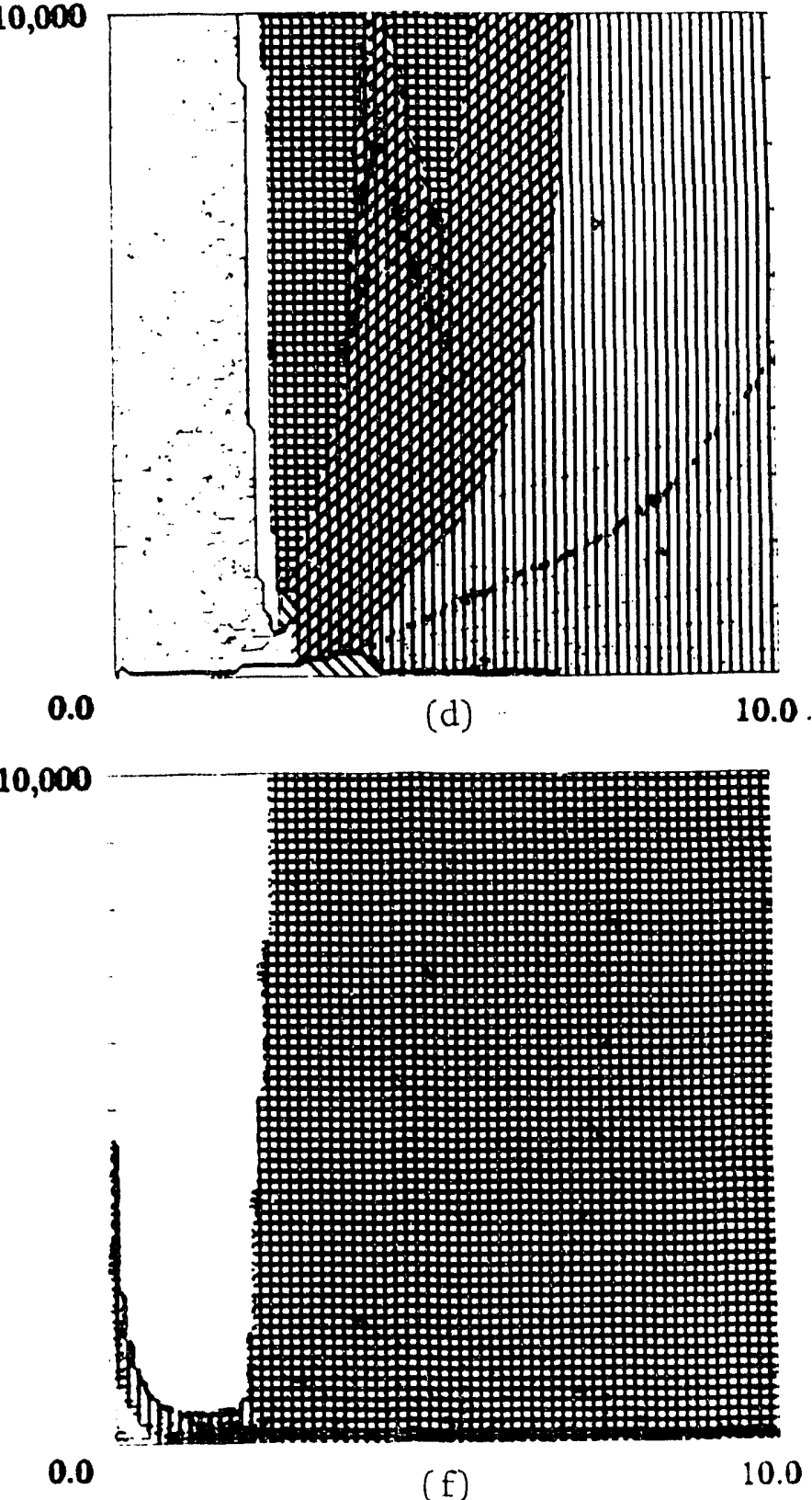

Fig. 19 
PRANDEL *

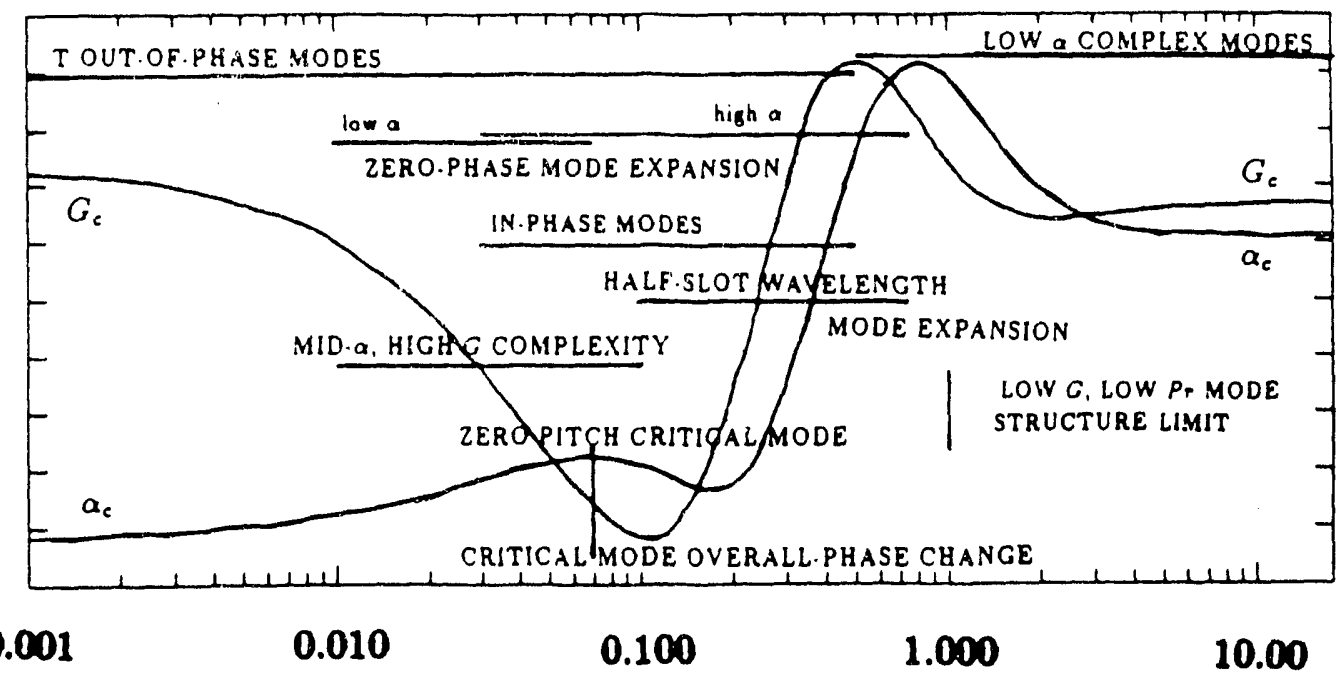

Fig. 20 

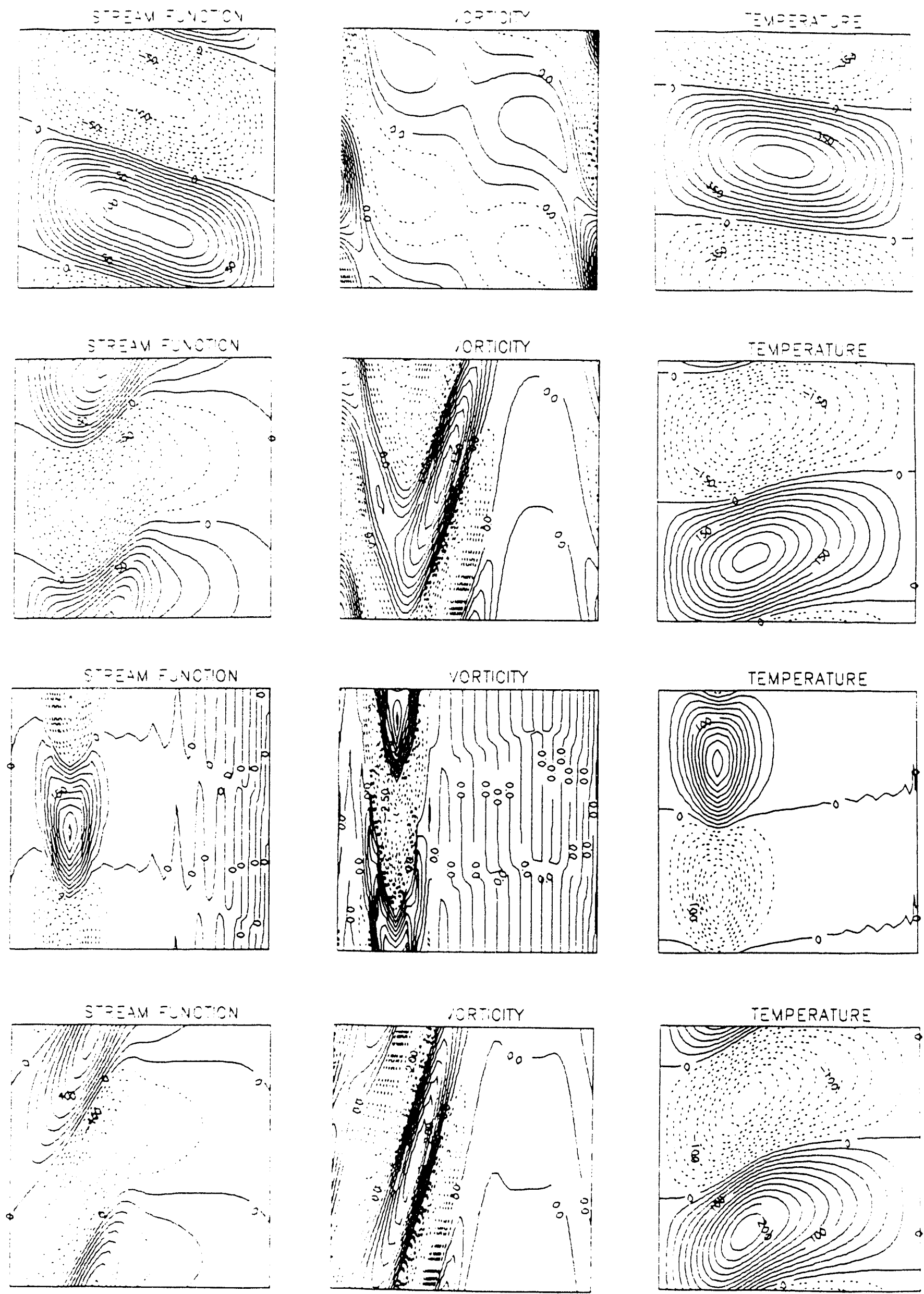

Fig. 21 

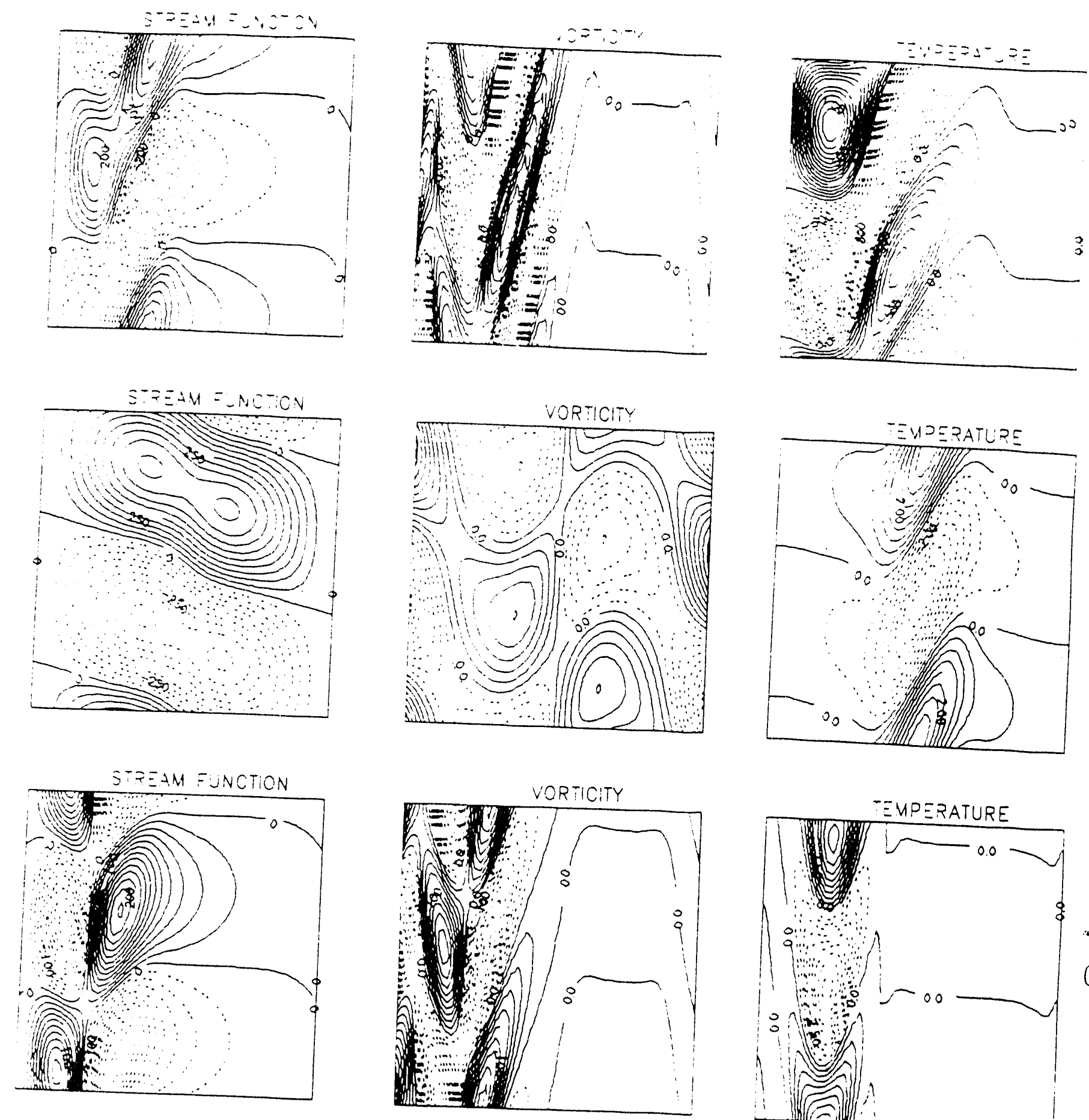

(b)
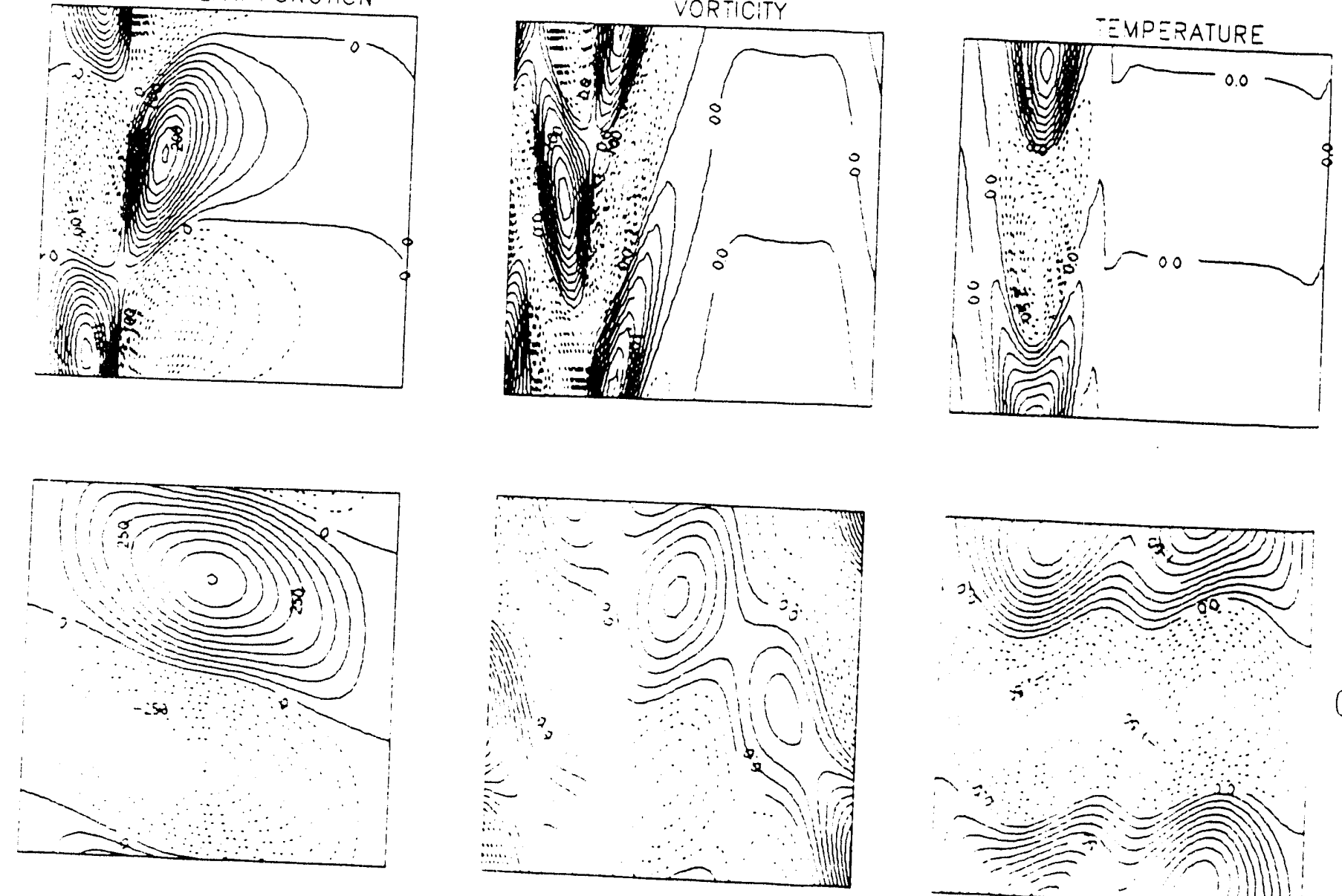

(c)

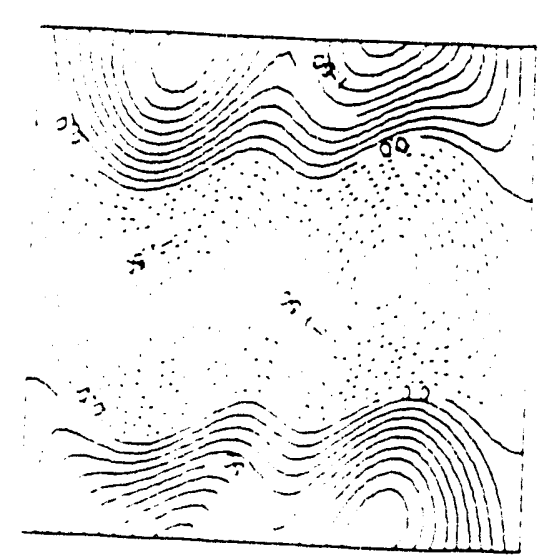

Fig. 22 

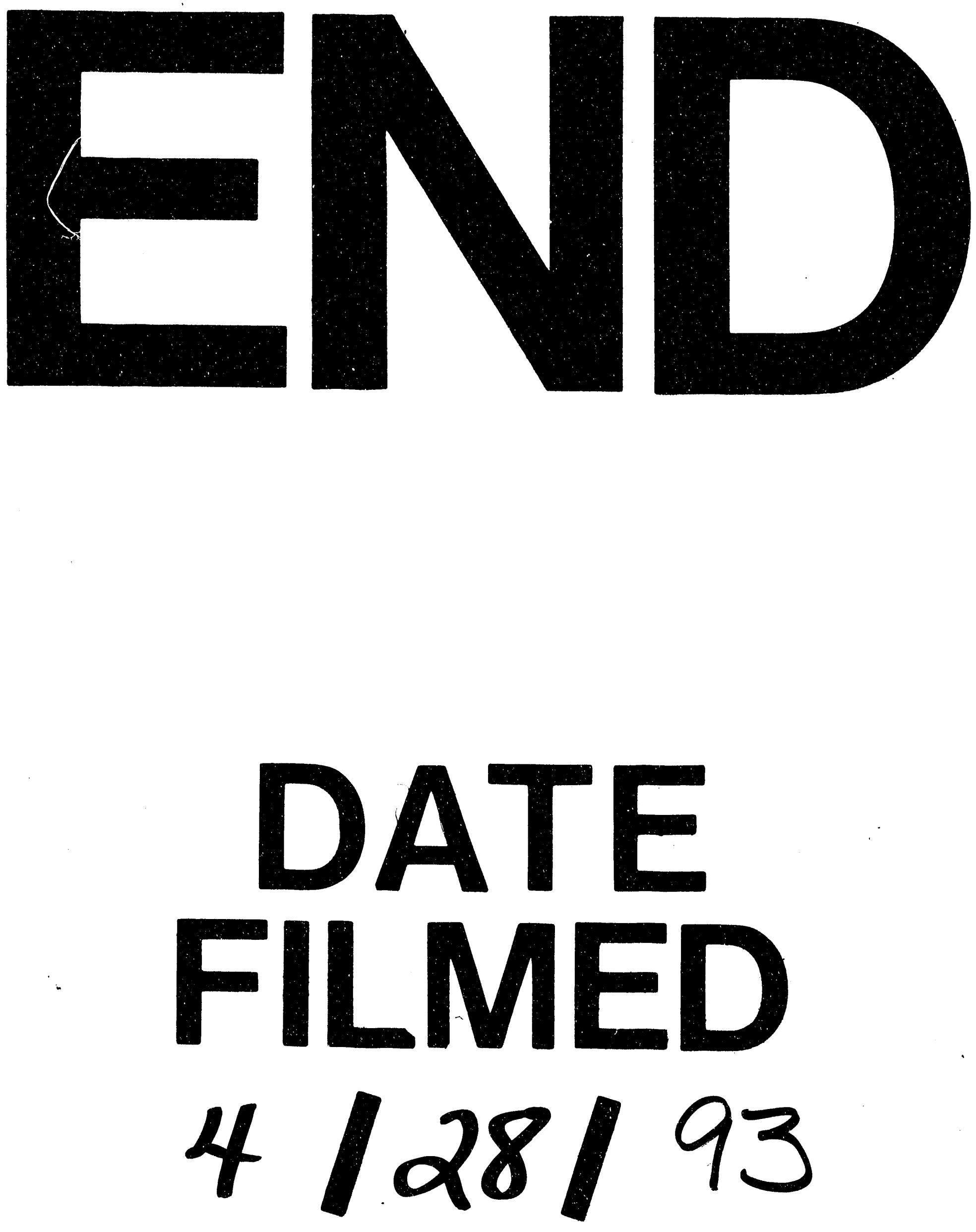

1 
